\title{
DRIVER RESTRAINT SYSTEM OPTIMIZATION FOR VEHICLE FRONTAL IMPACT
}

\author{
Anderson de Lima ${ }^{1}$, Eduardo L. Almeida ${ }^{1}$ e Marco A. Gouvea ${ }^{1}$ \\ ${ }^{1}$ General Motors Brazil
}

andersonkaipers.lima@gm.com, eduardo.almeida@gm.com, $\underline{\text { marco.gouvea@gm.com }}$

\begin{abstract}
In 2010, the UN General Assembly proclaimed the period 2011-2020 as the Decade of Action for Road Safety, with a goal to stabilize and then reduce the forecast level of road traffic fatalities around the world.
\end{abstract}

Road traffic accidents are the 8th cause of death in Brazil, according to World Health Rankings. There are few studies around the world with respect to cost due to traffic accidents, however a study performed in 2011 estimates that were spent $\mathrm{R} \$ 44.6$ billion in Brazil. So, the recent Brazilian regulations updates have enforced the automakers to develop vehicles safer to passengers and pedestrians. These regulations focus on prevent, reduce or minimize the traumas and injuries caused by different types of vehicular accidents.

The present work was developed to optimize the driver restraint system, while focusing on minimizing the trauma during a vehicle frontal impact. The driver restraint system was optimized considering the complex interaction between the ATD and the different components that assemble the restraint system, like airbag, safety belts with/without pretensioners, seatbelt load limiting devices and steering column stroke.

The numerical computational simulations were performed based on Design of Experiments (DOE), which is a powerful tool that allows for multiple input factors to be manipulated determining their effect on a desired output. The numerical computational model created was initially correlated with a physical test, and then 36 numerical simulations were performed in order to create the optimization matrix.

The optimized parameters provided by the analysis of the DOE orthogonal matrix were simulated and showed a significant reduction at probability of injuries due to vehicle frontal impact. The computational numerical optimization tool helped to reduce the cost and time development of a safer vehicle that satisfies the current Brazilian regulations, focusing on driver performance. The results presented excellent correlation and the goals of the optimization were achieved showing that this tool is reliable and helpful for current and future developments.

\section{INTRODUCTION}

In a frontal impact collision, the restraint system helps reduce the risk of traumas and injuries of the occupants caused by occupant contact with the vehicle interior. 
The secondary impact in a collision is between the occupant and the restraint system and/or the vehicle interior. So, frontal restraint system plays an important role in the secondary impact reducing the occupant's excursion in the vehicle interior preventing their contact with instrument panel and/or steering wheel.

The restraint system is a set of devices which can be compound by seatbelt with and without load limiter, airbags, retractor with and without pre-tensioner, etc, see Figure 1. Also, steering column collapsible helps minimize the hard contact between the occupant and the steering wheel.
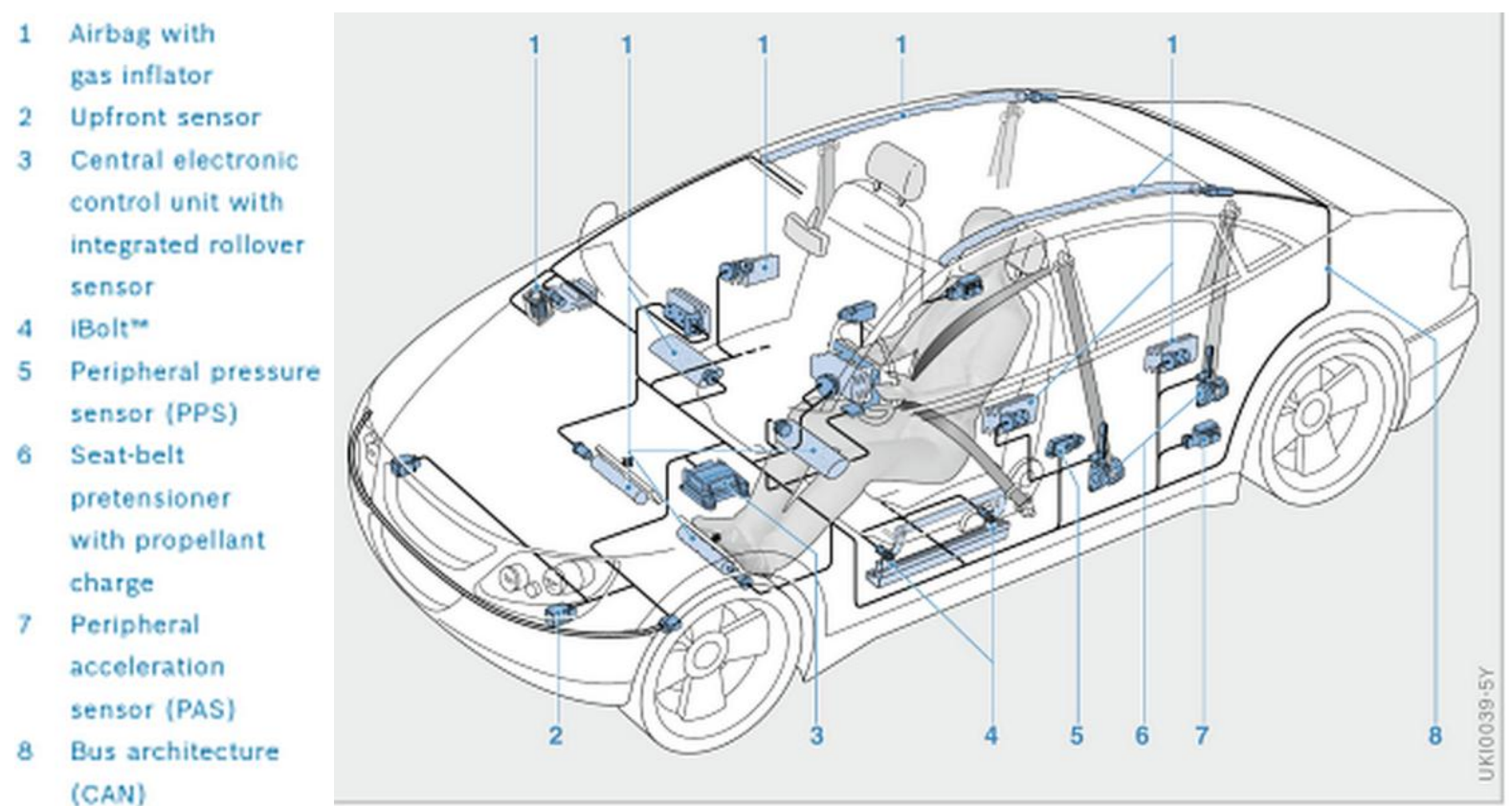

Figure 1. Example of vehicle restraint systems components [1].

The recent Brazilian regulations updates have enforced the automakers to develop vehicles safer to passengers and pedestrians. These regulations focus on prevent, reduce or minimize the traumas and injuries caused by different types of vehicular accidents.

The main focus of this work was to optimize the restraint system of a current vehicle in production using Design For Six Sigma (DFSS) optimization tools based on Design of Experiments (DOE). DFSS is a proactive business process utilizing the voice of the customer into the design of products and processes. The methodology applied helped to develop a robust restraint system in order to achieve the CONTRAN Resolution No. 221/07 with robust safety margin.

The present paper only focused on the driver's restraint system optimization. The optimization was developed considering the vehicle frontal impact at $56 \mathrm{~km} / \mathrm{h}$ against a deformable barrier at $40 \%$ overlap (ODB) in accordance with the ABNT NBR 15300 option 3 standard and test procedure which is based on Economic Commission Europe (ECE) R94 standard. The test shall be carried out with two 50th percentile male Hybrid III dummies belted at front seats. Figure 2 illustrates test setup. 

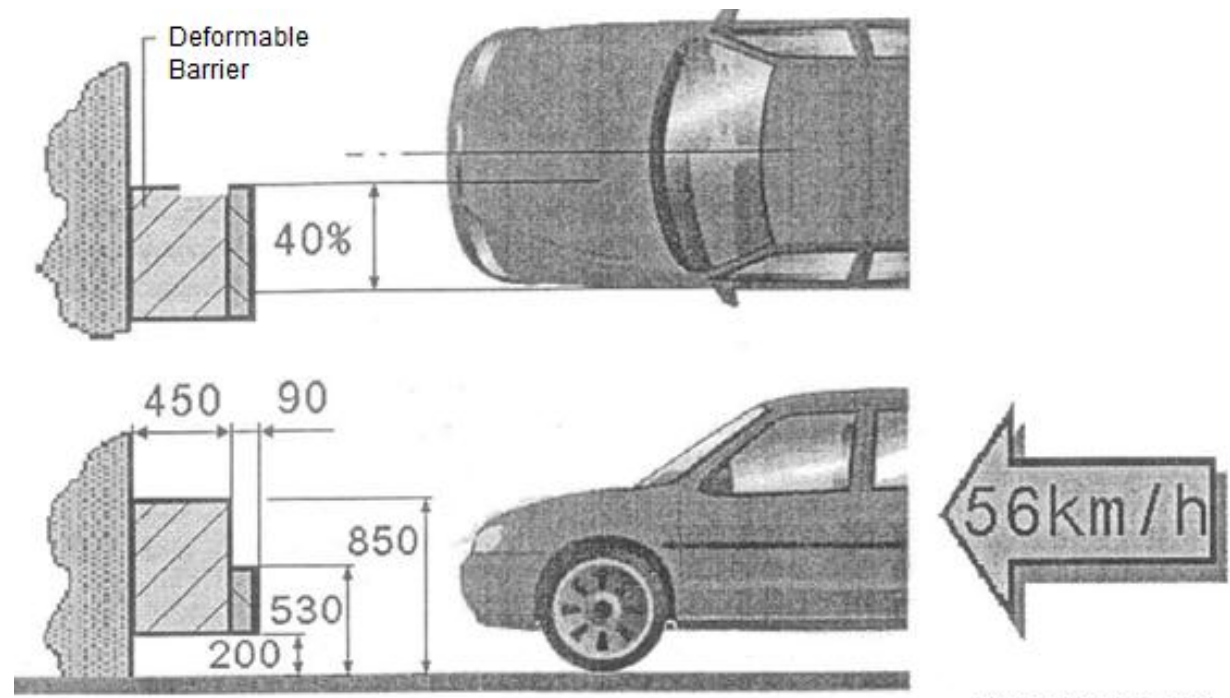

Dimensions in $\mathrm{mm}$

Figure 2. ABNT NBR 15300-3 barrier test setup.

The vehicles with the test dummies must comply with the occupant protection requirements according to CONTRAN 221/07 (ABNT NBR 15300-3 - Road vehicles - Occupant protection - Frontal crash test Part 3: frontal crash test procedure with $40 \%$ overlap).

The study was approached through the IDDOV process in DFSS methodology. By following the IDDOV framework a product is designed around the customer needs and wants. IDDOV in DFSS provides the necessary framework for product development and emphasizes the stepby-step phases to achieve Six Sigma, including Identify, Define, Develop, Optimize and Verify. Figure 3 shows the five steps of IDDOV process.

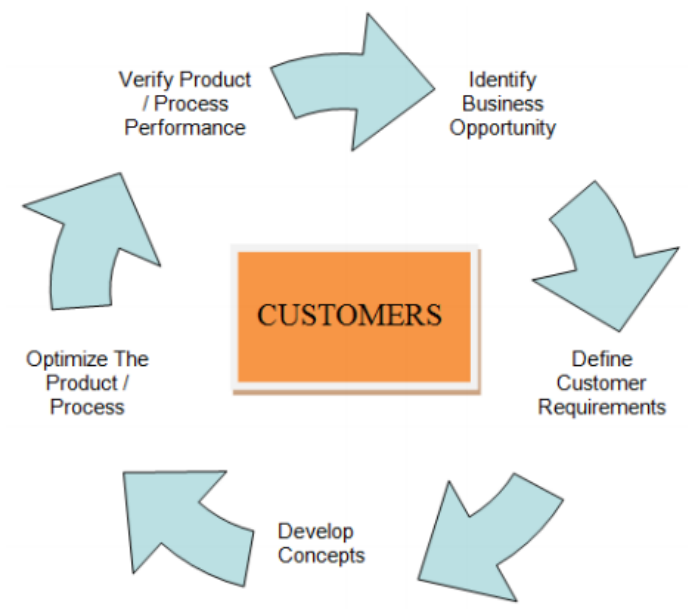

Figure 3. IDDOV five steps [2].

After establish the control factors levels and noise factors, an orthogonal array of experiments is selected to perform the DOE. By basing the experiments on an orthogonal array it is ensured that all possible combinations and levels occur together equally often. Control factors are any design parameters of a system that engineers can specify their nominal values for and maintain cost effectively and noise factors are sources of uncontrollable variation that affect a systems function [2]. 
In order to optimize the driver's restraint system a set of numerical computational simulations were performed based on Design of Experiments (DOE), which is a powerful tool that allows for multiple input factors to be manipulated determining their effect on a desired output. The numerical computational model created was initially correlated with a physical test, and then 36 numerical simulations were performed in order to create the optimization matrix.

\section{METHODOLOGY}

\subsection{IDDOV - Identify Phase}

In this phase is that an opportunity is identified. It is necessary to identify the customer requirements, their needs are prioritized and translate to measured requirements. This phase is probably the most important stage in any DFSS process, and the time spent here will result in a much better final project. The benefit for the customer is to improve the occupant protection by reducing potential injuries during a frontal impact.

The assessment of physical crash test performed with vehicle presented the opportunity was to modify the current DAB (Driver AirBag), load limiter and steering column stroke to reduce the complexity of seatbelt retractor canceling the driver safety belt pre-tensioner but keeping the occupant injury safety margin for regulatory requirement.

Several variables were considered during the work and its importance will be explored in details throughout the project. The design variables were: airbag vent hole diameter, airbag with and without tethers varying length and position, load limiter of seatbelt and seatbelt height adjustment. Some components, like the seat travel and design, instrument panel design, steering wheel design position which are carry-over from the current vehicle and will not be modified for this work. Figure 4 shows the project scope.

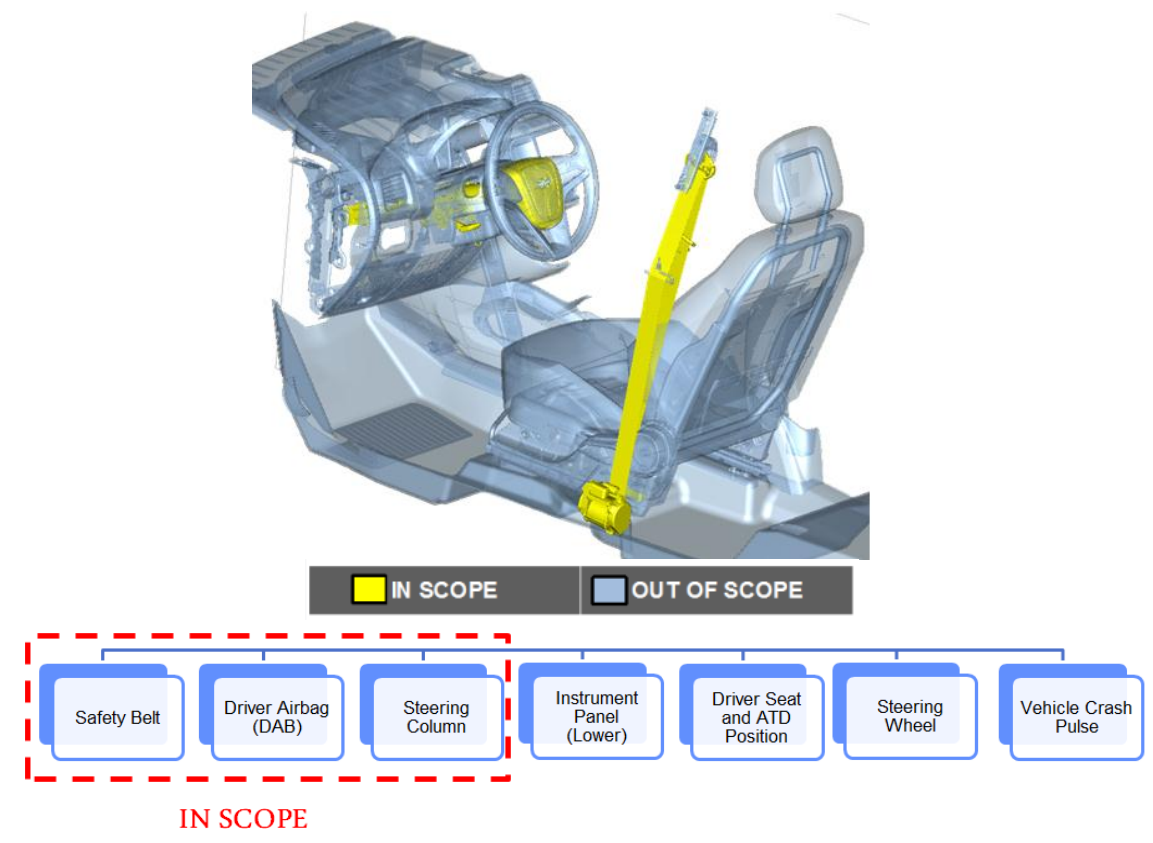

Figure 4. Project scope. 


\subsection{IDDOV - Define Requirements}

For this phase the needs and the desires of the customers are translated into specific product requirements. The voice of clients is:

- Safer car to drive;

- Occupant protected from serious injuries during frontal collision events.

These voices should be translated in measured metrics, in that case the vehicles must fulfill the Brazilian regulation based on CONTRAN 221/2007 Legal Requirement (Occupant Protection) which specify: the vehicles with the test dummies must comply with the occupant protection requirements listed on Table 1 below according CONTRAN 221/07 (ABNT NBR 15300-3 - Road vehicles - Occupant protection - Frontal crash test Part 3: frontal crash test procedure with $40 \%$ overlap). After the impact event, the dummy's biomechanical responses are assessed in six distinct body regions, as shown in the Figure 5.

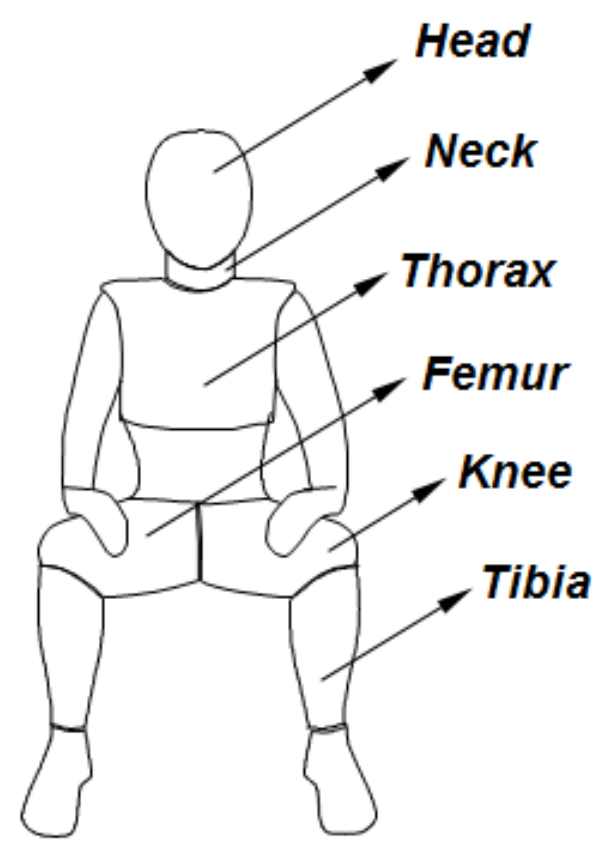

Figure 5. Body regions to injuries assess.

Table 1 shows the injuries criteria and their maximum acceptable values with respect to 50th percentile male Hybrid III dummies in accordance with CONTRAN Resolution No 221/2007 that establishes the legal requirements focusing on occupant protection. 
Table 1. CONTRAN 221/07 Performance Requirements.

\begin{tabular}{|c|c|c|c|}
\hline \multirow{2}{*}{ Dummy Region } & Injury Criteria & Units & Values \\
\hline \multirow{2}{*}{ Head } & HIC (36ms) & {$[-]$} & 1000 \\
\cline { 2 - 4 } & Resultant Acceleration (3ms) & {$[\mathrm{g}]$} & 80 \\
\hline \multirow{4}{*}{ Neck } & Axial Tension (+Fz) - Duration & {$[\mathrm{N}]$} & $3300(0 \mathrm{~ms})$ \\
\cline { 2 - 4 } & & & $2900(35 \mathrm{~ms})$ \\
\cline { 2 - 4 } & Shear Load (+Fx) - Duration & {$[\mathrm{N}]$} & $100(\geq 60 \mathrm{~ms})$ \\
\cline { 2 - 4 } & & & $1500(25-35 \mathrm{~ms})$ \\
\cline { 2 - 4 } & Rearward Moment - Ext (-My) & {$[\mathrm{Nm}]$} & $1100(>45 \mathrm{~ms})$ \\
\cline { 2 - 4 } & Compression (Belted) & {$[\mathrm{mm}]$} & 57 \\
\hline \multirow{2}{*}{ Thorax } & Viscous Criterion & {$[\mathrm{m} / \mathrm{s}]$} & 1.0 \\
\hline \multirow{2}{*}{ Femur } & Compressive Load - Duration & {$[\mathrm{N}]$} & $9070(0 \mathrm{~ms})$ \\
\hline \multirow{2}{*}{ Tibia } & Axial Compressive Load & {$[\mathrm{N}]$} & $7580(10 \mathrm{~ms})$ \\
\cline { 2 - 4 } & Index= M/Mc + |P/Pc & {$[-]$} & 1.3 \\
\hline \multirow{2}{*}{ Knee } & Sliding (Tibia-Femur Translation) & {$[\mathrm{mm}]$} & 15 \\
\hline
\end{tabular}

Project main focus is chest compression, but all CONTRAN 221/07 injury levels must be met, since it's a Brazilian legal requirement.

\subsection{IDDOV - Develop / Design Phase}

In this stage, the DFSS process takes a feasible turn. Here are generated conceptual proposals and it is select the best concept design and technology. The concept may be developed at several levels starting with the system architecture for the entire product. Then, concepts are developed for the various system elements as needed [3].

To meet the Driver chest compression requirement with Safety Belt without pretensioner is required balance the energy absorbed by tune of:

- Steering Column (crush load)

- Driver Airbag (hardness)

- Safety Belt Load limiter.

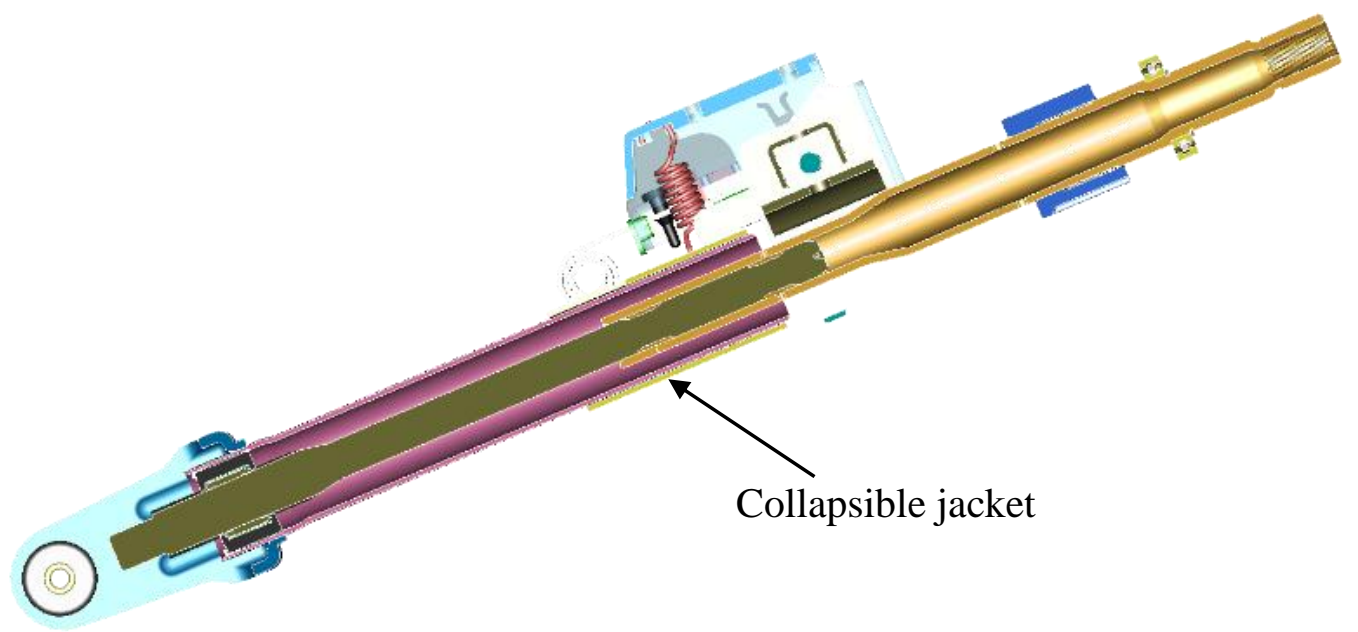

Figure 6. Steering column collapsible. 


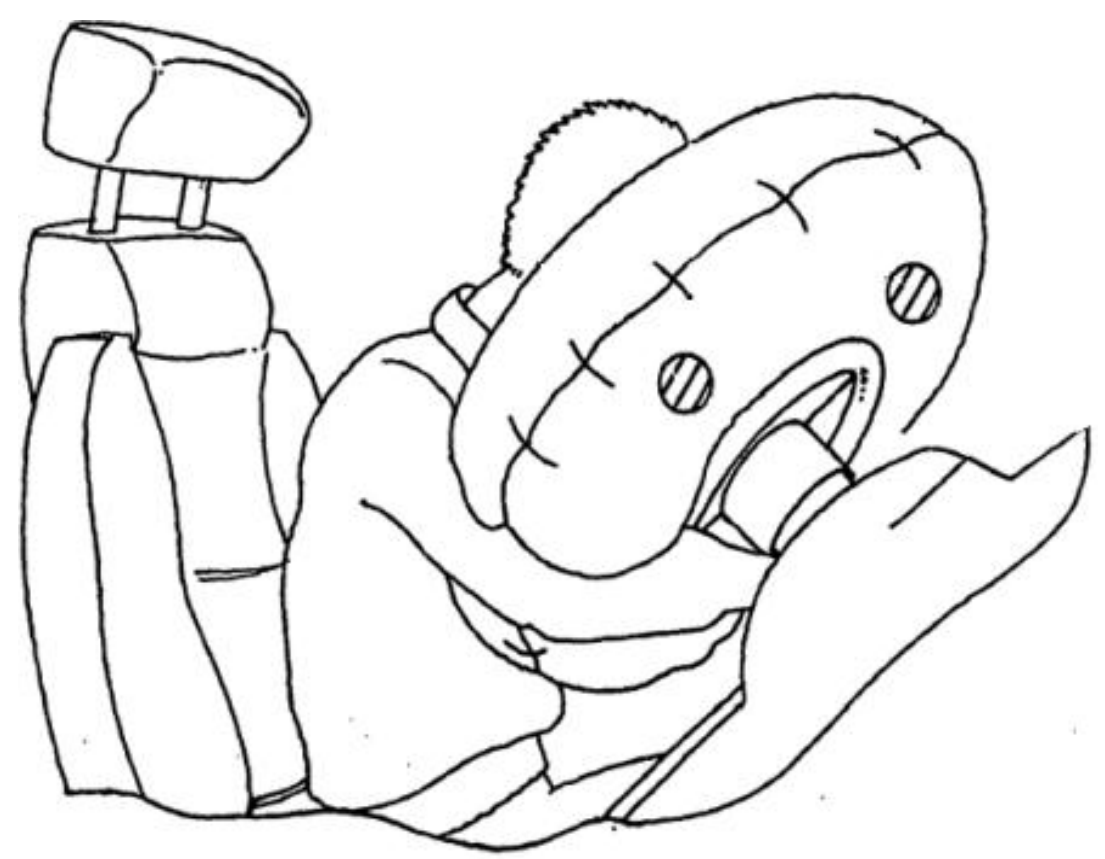

Figure 7. Driver Airbag.

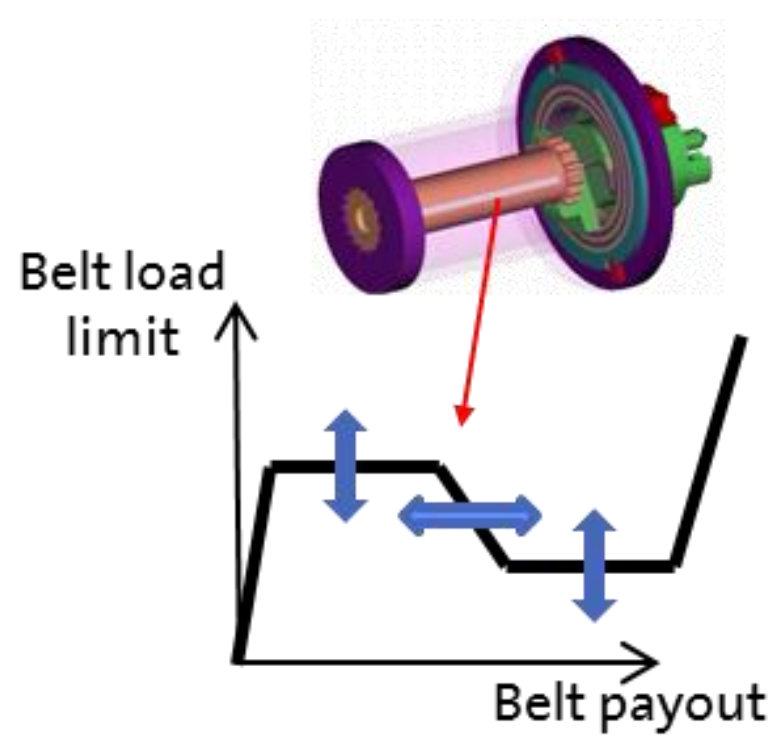

Figure 8. Load limiter.

For the driver airbag, 2 concepts were defined. Figure 9 illustrates the airbag concepts for this project with and without tether. For airbags with tether was varied its position and length. Figure 10. 


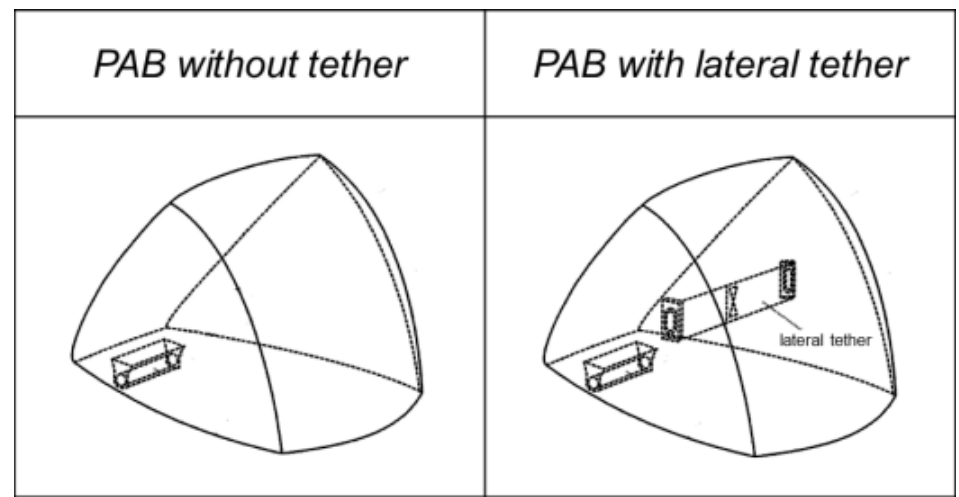

Figure 9. Airbag concept selection.

\section{Tether type \& position}

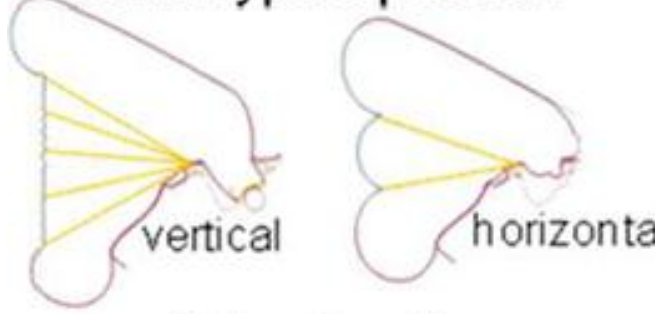

Tether length

short

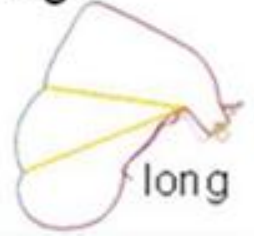

Figure 10. Tether configurations.

\subsection{IDD弚 - Optimize Phase}

This phase focuses in develop the product to be robust. Robustness is defined as "the state of performance where the technology, product, or process is minimally sensitive to factors causing variability (either in the manufacturing or end users environment) at the lowest possible cost" [2].

Robust optimization uses orthogonal arrays to develop efficient Design of Experiments that are balanced. The Optimize Phase begins by conducting the eight step parameter design process:

1. Define Scope for Optimization

2. Identify Ideal Function / Response

3. Develop Signal and Noise Factor Strategies

4. Establish Control Factors and Levels

5. Execute and Control Data

6. Conduct Data Analysis

7. Predict and Confirm

8. Document and Verify 


\section{Define Scope for Optimization}

The project scope is to develop a driver restraint system with the lowest chest compression value as possible, which is able to meet all injuries criteria limits established by CONTRAN. A system diagram (showed in Figure 11) is useful for describing the scope.

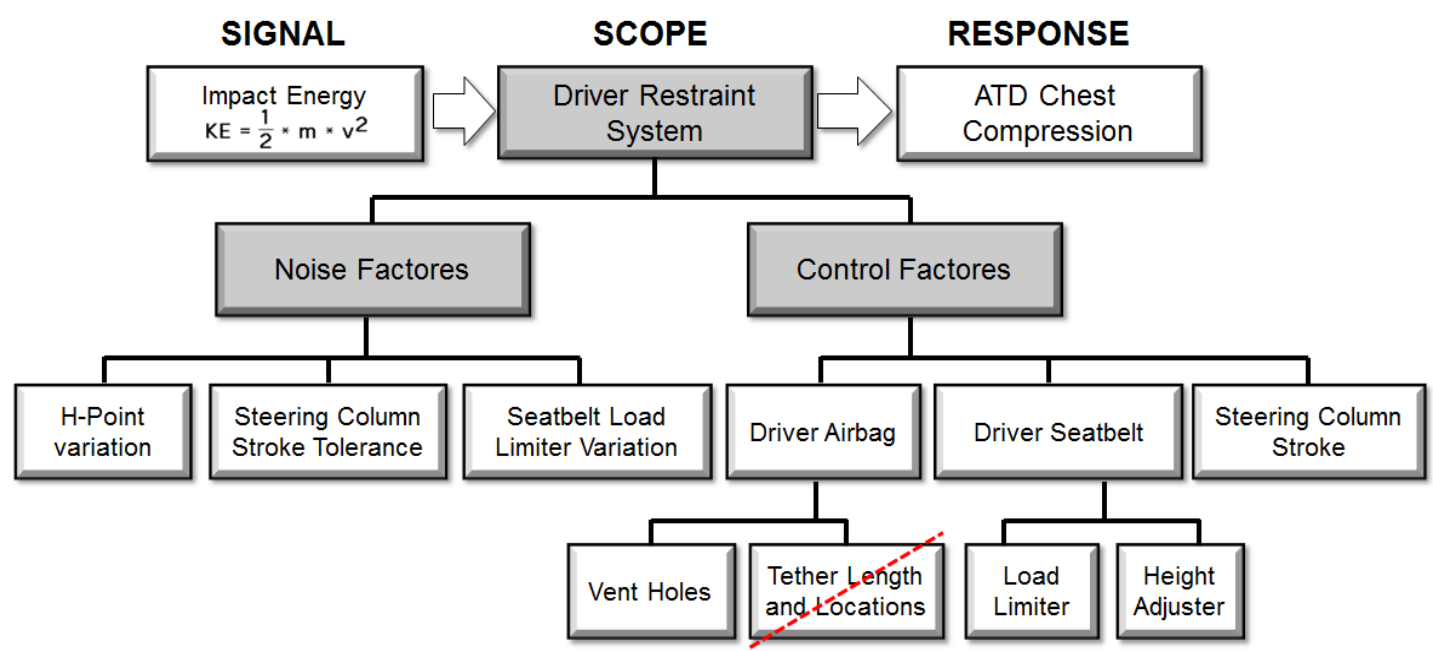

Figure 11. System diagram.

The red dashed control factor was considered or not during the optimization phase.

\section{Identify Ideal Function / Response}

The driver's restraint system should manage and absorb the appropriate level of energy which was not absorbed by the vehicle structure, in a short period of time. The loads passing through the restraint system should be balanced in order to provide uniform loading distribution in the occupant. As our main target in the optimization is the chest compression, the chest deformation energy will be the response of the ideal function and its minimization or maintenance is the principal goal. The chest compression energy is function of the chest displacement and the force.

$$
E_{\text {def }}=\text { Chest displacement } \times \text { Chest load }
$$

\section{Develop Signal and Noise Factor Strategies}

The $\mathrm{S} / \mathrm{N}$ ratio functions as a single measure of robustness and a gain in the $\mathrm{S} / \mathrm{N}$ ratio reflects an improvement in robustness [4]. For each experiment a $\mathrm{S} / \mathrm{N}$ and $\beta$ will be calculated. The $\mathrm{S} / \mathrm{N}$ is an index of robustness. The higher the $\mathrm{S} / \mathrm{N}$, the more the system is doing what it is intended to do. It measures the quality of energy transformation that occurs within a design. As the input signal, energy transformation, output response, and noise factors come together, their combined effect creates the design's $\mathrm{S} / \mathrm{N}$. The slope of the output response $(\beta)$ is the sensitivity of the input signal to the output response.

$$
\mathrm{Y}(\text { output response })=\beta \mathrm{M} \text { (input signal })
$$


In general, the $\mathrm{S} / \mathrm{N}$ ratio for quality characteristics is based on mean squared deviation (MSD) and can be calculated as given in equation below.

$$
\mathrm{S} / \mathrm{N}=-10 \log 10(\mathrm{MSD})
$$

\section{Signal levels}

The signal for this study was based on the worst case for physical test pulse. The pulse was gotten from accelerometer located at rocker in the same side of the impact.

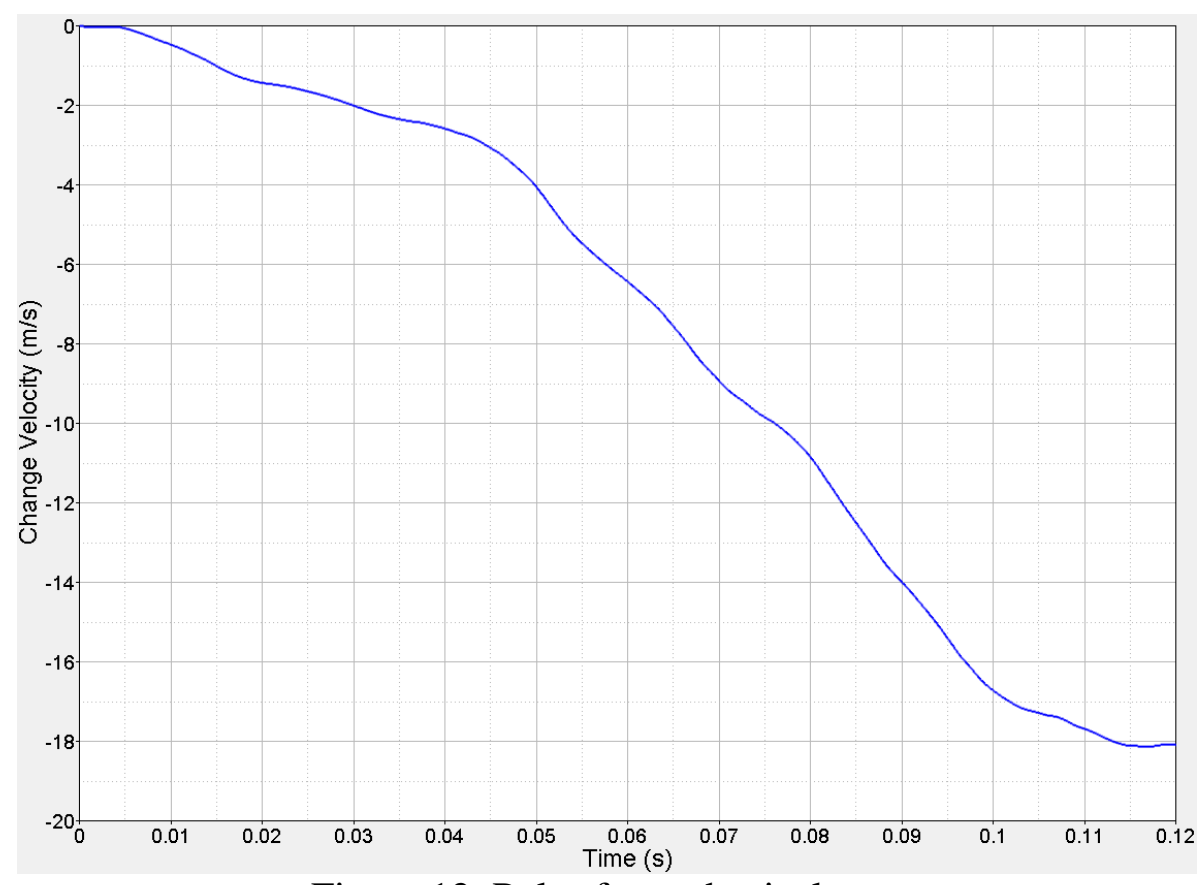

Figure 12. Pulse from physical test.

\section{Noise factors}

The present study considers three noise factors which were chosen considering the influence in the output response: H-point variations (X/Z directions), seatbelt load limiter variation and steering column stroke tolerance. Such as control factors levels, these noise factors can be evaluated by computational numerical simulation. According to ABNT NBR 15300 option 1, the H-point tolerance is $13 \mathrm{~mm}$, however based on previously physical tests performed the tolerance verified was $9 \mathrm{~mm}$ with respect to design (nominal) position. Figure 13 shows H-point variation.

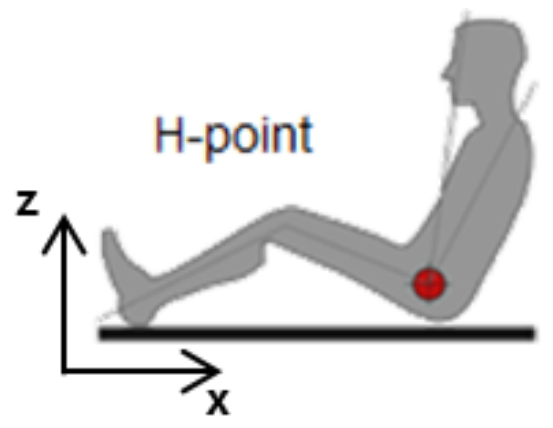




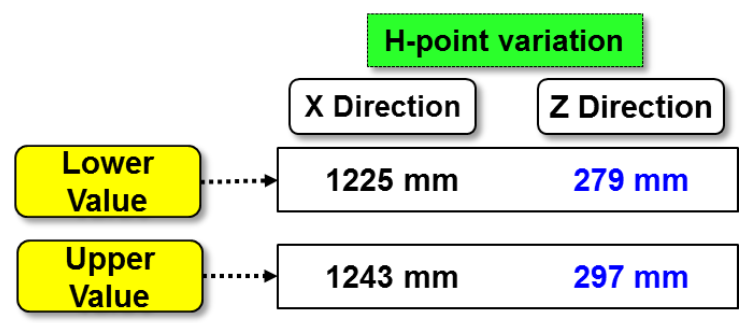

Figure 13. H-point variations.

The load limiter presents variation of $+0.5 \mathrm{kN}$ during its activation. So, numerical simulations were performed varying driver seatbelt loads considering this range to understand which can affect chest compression results due to this variation. It was evaluated load limiters with three nominal loads. Figure 14 shows the three different load limiter values.

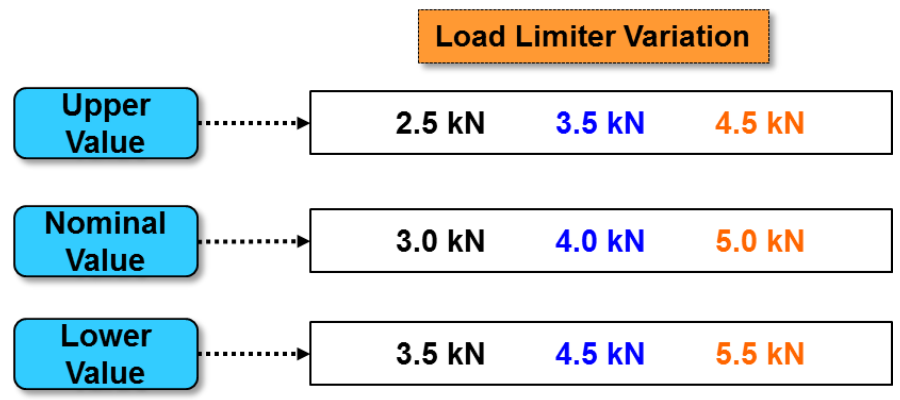

Figure 14. Load limiter variations.

According to steering column supplier there are tolerances in the initial peak load and also in the running load. Its variance is $\pm 700 \mathrm{~N}$ and $\pm 1000 \mathrm{~N}$, respectively. During the DFSS project we proposed study 2 different steering column collapse load, in addition of the nominal collapse load used in the vehicle in production. For the additional steering columns proposal were considered the same tolerances of the current steering column. Figure 15 shows the steering column loads tolerance.

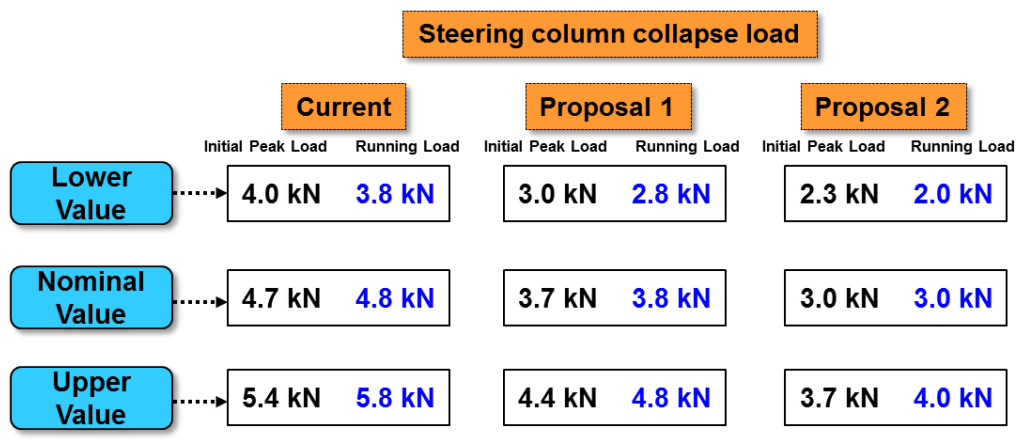

Figure 15. Steering column collapse load variations.

\section{Establish Control Factors and Levels}

The engineering design parameters are the control factors. In the present work five control factors were evaluated during the optimization phase which are expected to influence the relationship between the input and output. 
1. Seatbelt Load limiter. The three levels specified for this control factor are: 3.0 $\mathrm{kN}, 4.0 \mathrm{kN}$ and $5.0 \mathrm{kN}$.

2. Driver Airbag Vent Holes Diameter. The three levels specified for this control factor are $2 \times 15 \mathrm{~mm}, 2 \times 25 \mathrm{~mm}$ and $2 \times 35 \mathrm{~mm}$.

3. Driver Airbag Tethers. The three levels specified for this control factor considering length, position and without tether are:

Level 1: Position 3h \& 9h with length of $338 \mathrm{~mm}$;

Level 2: Position $3 \mathrm{~h} \& 9 \mathrm{~h}$ with length of $338 \mathrm{~mm}+$ Position $6 \mathrm{~h}$ with length of $332 \mathrm{~mm}$ );

Level 3: No tether.

4. Seat belt height adjuster. The three levels specified for the seat belt height adjuster are: lower, medium and upper positions.

5. Steering column stroke. This control factor was evaluated considering three sets of initial peak load and running load as:

Level 1: Initial peak load $4.7 \mathrm{kN}$, Running load $4.8 \mathrm{kN}$;

Level 2: Initial peak load $3.7 \mathrm{kN}$, Running load $3.8 \mathrm{kN}$;

Level 3: Initial peak load $3.0 \mathrm{kN}$, Running load $3.0 \mathrm{kN}$.

\section{Parameter Diagram}

The P-diagram, or parameter diagram, provides a convenient and orderly way to organize and display control factors, noise factors, input signal, and output response [2]. Figure 16 illustrates the P-Diagram for the project.

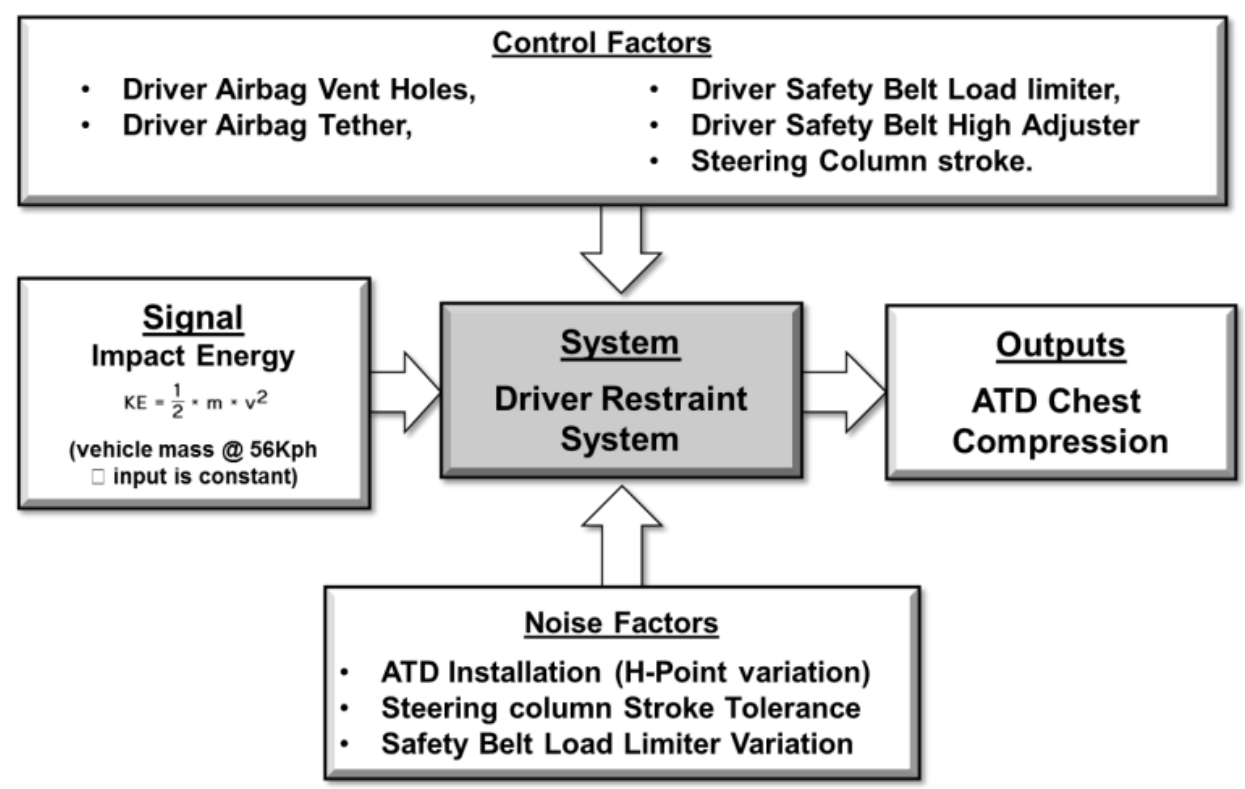

Figure 16. P-Diagram.

\section{Execute and Control Data}

During the 1950's Dr. Genichi Taguchi developed a methodology similar to how the DFSS process is used today. Dr. Taguchi's use of system thinking, approach to DOE's using orthogonal arrays, Quality Loss Function, and using Signal to Noise Ratio in Robust Optimization are unique to the DFSS process [2]. 
The design of experiment (DOE) method has been widely used by industries to improve the quality of product. With the help of this technique, the effect of several variables could be studied efficiently. The objective of the experiment is to make comparisons between the effects of different factors and then determine the best setting for each factor [5].

The present work used 5 control factors at 3 levels and 3 noise factors, as shown in Figure 17.

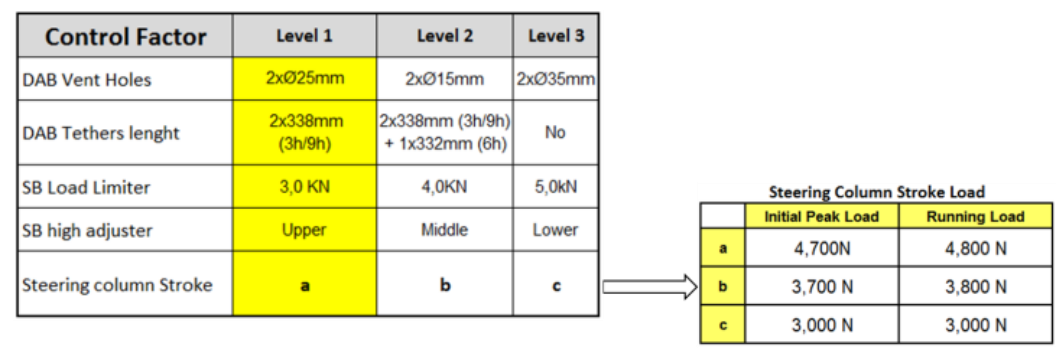

\begin{tabular}{|l|c|c|}
\hline \multicolumn{1}{|c|}{ Noise Factor } & N1 & N2 \\
\hline ATD Position (H point x/z) & $\begin{array}{c}1225 / 297 \\
\text { (Up Front) }\end{array}$ & $\begin{array}{c}1243 / 279 \\
\text { (Down Rear) }\end{array}$ \\
\hline Load Limiter Tolerance & $\begin{array}{c}\text { Nominal } \\
-0,5 ~ N\end{array}$ & $\begin{array}{c}\text { Nominal } \\
+0,5 \mathrm{~N}\end{array}$ \\
\hline Steering column Stroke Tol. & $+700 \mathrm{~N} /$ & $-700 \mathrm{~N} /$ \\
(lnicial Peak Load / Running Load) & $+1000 \mathrm{~N}$ & $-1000 \mathrm{~N}$ \\
\hline
\end{tabular}

Figure 17. Control and Noise Factors.

The team selected the popular L18 orthogonal array that allows up to 7 control factors with 3 levels variation and 1 control factor with 2 levels variation.

In order to reduce the total time of numerical simulation of a full vehicle colliding against a deformable barrier, the computational numerical simulations were performed simulating a sled test. Firstly, the finite elements model was correlated with physical tests. Figure 18 shows the good correlation between numerical model and physical tests conducted with and without pre-tensioner.

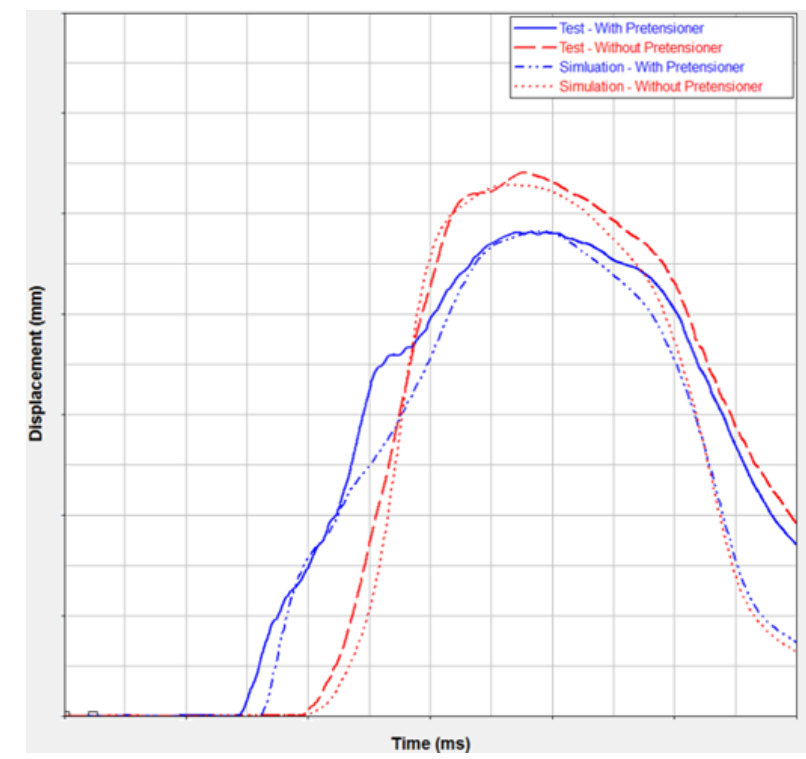

Figure 18. Correlation between finite elements model and physical test. 
The computational simulations were performed using the MADYMO software [6]. MADYMO is a powerful computer program that simulates the dynamic behavior of physical systems.

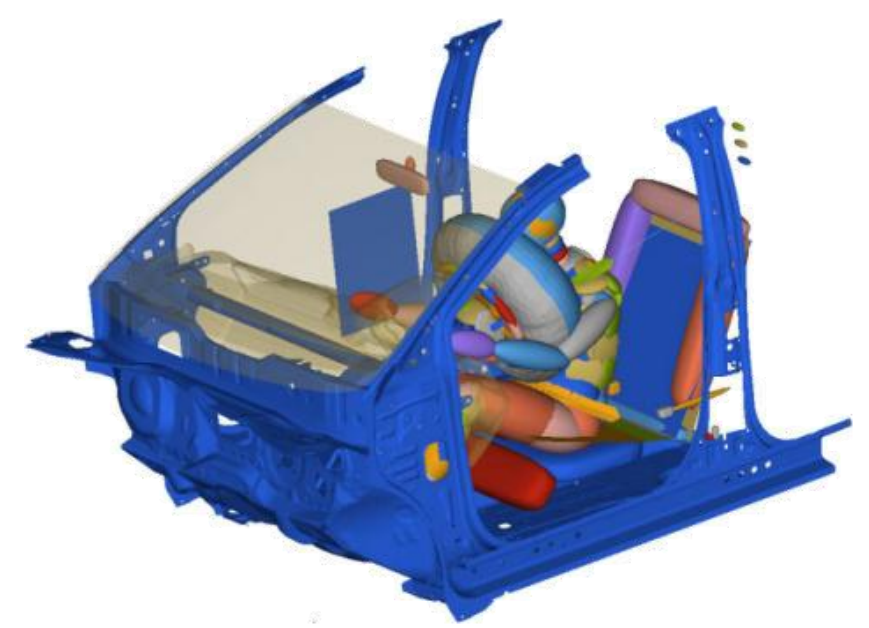

Figure 19. Finite elements model to simulate at MADYMO.

The control factors and noise factors were combined in the L18 orthogonal array that generated a Design Of Experiments (DOE) matrix that provided data to run 36 numerical simulations (CAE assessment) as shown on Table 2.

Table 2. DOE matrix

\begin{tabular}{|c|c|c|c|c|c|c|c|c|}
\hline & \multicolumn{5}{|c|}{ CONTROL FACTORS } & \multicolumn{3}{|c|}{ Noise Factor } \\
\hline & $\begin{array}{c}\text { A } \\
\text { DAB Vent Hole }\end{array}$ & $\begin{array}{c}\text { B } \\
\text { DAB Tether }\end{array}$ & $\begin{array}{c}\text { C } \\
\text { SB Load } \\
\text { Limiter }\end{array}$ & $\begin{array}{c}\text { D } \\
\text { SB High Adj. }\end{array}$ & $\begin{array}{c}\text { E } \\
\text { S. Column } \\
\text { Stroke }\end{array}$ & $\begin{array}{c}\text { ATD } \\
\text { Position (H } \\
\text { point } x / z)\end{array}$ & $\begin{array}{c}\text { Load } \\
\text { Limiter } \\
\text { Tolerance }\end{array}$ & $\begin{array}{c}\text { St. Column } \\
\text { Stroke Tol. } \\
\text { (Inicial Peak } \\
\text { Load / Running } \\
\text { Load)) }\end{array}$ \\
\hline Simulation 1 & \begin{tabular}{|c|}
1 -DAB Vent \\
Holes $2 \times \varnothing 25 \mathrm{~mm}$
\end{tabular} & $\begin{array}{c}1 \text { - DAB Tethers } 2 \times 338 \mathrm{~mm} \\
(3 \mathrm{~h} / 9 \mathrm{~h})\end{array}$ & $\begin{array}{c}1 \text { - SB LL } 3,0 \\
\mathrm{kN}\end{array}$ & $\begin{array}{c}1 \text { - SB H. Adj. } \\
\text { Upper }\end{array}$ & $\begin{array}{c}1 \text { - SC Stroke } \\
\text { "a" }\end{array}$ & & & \\
\hline Simulation 2 & \begin{tabular}{|c|}
1 -DAB Vent \\
Holes $2 \times \varnothing 25 \mathrm{~mm}$
\end{tabular} & $\begin{array}{c}\text { 1- DAB Tethers } 2 \times 338 \mathrm{~mm} \\
(3 \mathrm{~h} / 9 \mathrm{~h})\end{array}$ & $\begin{array}{c}1-\mathrm{SB} \text { LL } 3,0 \\
\mathrm{kN}\end{array}$ & $\begin{array}{c}1 \text { - SB H. Adj. } \\
\text { Upper }\end{array}$ & $\begin{array}{c}1 \text { - SC Stroke } \\
\text { "a" }\end{array}$ & $\begin{array}{l}1243 / 279 \\
\text { (Down Rear) }\end{array}$ & $\begin{array}{l}\text { Nominal } \\
+0,5 \mathrm{~N}\end{array}$ & $\begin{array}{l}-700 \mathrm{~N} / \\
-1000 \mathrm{~N} \\
\end{array}$ \\
\hline Simulation 3 & \begin{tabular}{|c|}
1 -DAB Vent \\
Holes $2 \times Ø 25 \mathrm{~mm}$
\end{tabular} & $\begin{array}{l}\text { 2- DAB Tethers } 2 \times 338 \mathrm{~mm} \\
(3 \mathrm{~h} / 9 \mathrm{~h})+1 \times 332 \mathrm{~mm}(6 \mathrm{~h})\end{array}$ & $\begin{array}{l}\text { 2- SB LL 4,0 } \\
\text { kN }\end{array}$ & $\begin{array}{l}\text { 2- SB H.Adj. } \\
\text { Middle }\end{array}$ & $\begin{array}{c}\text { 2-SC Stroke } \\
\text { "b" }\end{array}$ & $\begin{array}{l}1225 / 297 \\
\text { (Up Front) }\end{array}$ & $\begin{array}{c}\text { Nominal } \\
-0,5 \mathrm{~N}\end{array}$ & \\
\hline Simulation 4 & \begin{tabular}{|c|}
1 -DAB Vent \\
Holes $2 \times \varnothing 25 \mathrm{~mm}$ \\
\end{tabular} & $\begin{array}{c}\text { 2- DAB Tethers } 2 \times 338 \mathrm{~mm} \\
(3 \mathrm{~h} / 9 \mathrm{~h})+1 \times 332 \mathrm{~mm}(6 \mathrm{~h})\end{array}$ & $\begin{array}{c}\text { 2- SB LL 4,0 } \\
\mathrm{kN}\end{array}$ & $\begin{array}{l}\text { 2- SB H.Adj. } \\
\text { Middle }\end{array}$ & $\begin{array}{c}\text { 2- SC Stroke } \\
\text { "b" }\end{array}$ & $\begin{array}{l}1243 / 279 \\
\text { (Down Rear) }\end{array}$ & & \\
\hline Simulation 5 & $\begin{array}{c}\text { 1-DAB Vent } \\
\text { Holes } 2 \times \varnothing 25 \mathrm{~mm}\end{array}$ & 3- DAB No tether & $\begin{array}{c}3-\mathrm{SB} \text { LL } 3,0 \\
\mathrm{kN}\end{array}$ & $\begin{array}{l}3 \text { - SB H.Adj. } \\
\text { Lower }\end{array}$ & $\begin{array}{c}\text { 3- SC Stroke } \\
\text { "c" }\end{array}$ & $\begin{array}{l}1225 \text { / } 297 \\
\text { (Up Front) }\end{array}$ & $\begin{array}{c}\text { Nominal } \\
-0,5 \mathrm{~N}\end{array}$ & $\begin{array}{l}+700 \mathrm{~N} / \\
+1000 \mathrm{~N} \\
\end{array}$ \\
\hline Simulation 6 & \begin{tabular}{|c|}
1 -DAB Vent \\
Holes $2 \times \varnothing 25 \mathrm{~mm}$
\end{tabular} & 3- DAB No tether & $\begin{array}{l}3 \text { - SB LL } 3,0 \\
\mathrm{kN}\end{array}$ & $\begin{array}{c}\begin{array}{c}\text { 3 - SB H.Adj. } \\
\text { Lower }\end{array} \\
\end{array}$ & $\begin{array}{c}\text { 3- SC Stroke } \\
\text { "c" }\end{array}$ & $\begin{array}{c}1243 / 279 \\
\text { (Down Rear) } \\
\end{array}$ & & \\
\hline Simulation 7 & \begin{tabular}{|c|}
2 -DAB Vent \\
Holes $2 \times \varnothing 15 \mathrm{~mm}$
\end{tabular} & $\begin{array}{c}1 \text { - DAB Tethers } 2 \times 338 \mathrm{~mm} \\
(3 \mathrm{~h} / 9 \mathrm{~h})\end{array}$ & $\begin{array}{c}1-\mathrm{SB} \text { LL } 3,0 \\
\mathrm{kN}\end{array}$ & $\begin{array}{l}\text { 2- SB H.Adj. } \\
\text { Middle }\end{array}$ & $\begin{array}{c}\text { 2- SC Stroke } \\
\text { "b" }\end{array}$ & & $\begin{array}{c}\text { Nominal } \\
-0,5 \mathrm{~N}\end{array}$ & \\
\hline Simulation 8 & $\begin{array}{c}2 \text {-DAB Vent } \\
\text { Holes } 2 \times \varnothing 15 \mathrm{~mm}\end{array}$ & $\begin{array}{c}1 \text { - DAB Tethers } 2 \times 338 \mathrm{~mm} \\
(3 \mathrm{~h} / 9 \mathrm{~h})\end{array}$ & $\begin{array}{c}1-\mathrm{SB} \text { LL } 3,0 \\
\mathrm{kN}\end{array}$ & $\begin{array}{l}\text { 2- SB H.Adj. } \\
\text { Middle }\end{array}$ & $\begin{array}{c}\text { 2- SC Stroke } \\
\text { "b" }\end{array}$ & $\begin{array}{c}1243 / 279 \\
\text { (Down Rear) }\end{array}$ & $\begin{array}{l}\text { Nominal } \\
+0,5 \mathrm{~N}\end{array}$ & $\begin{array}{l}-700 \mathrm{~N} / \\
-1000 \mathrm{~N}\end{array}$ \\
\hline Simulation 9 & $\begin{array}{c}\text { 2-DAB Vent } \\
\text { Holes } 2 \times \varnothing 15 \mathrm{~mm}\end{array}$ & $\begin{array}{c}\text { 2- DAB Tethers } 2 \times 338 \mathrm{~mm} \\
(3 \mathrm{~h} / 9 \mathrm{~h})+1 \times 332 \mathrm{~mm}(6 \mathrm{~h})\end{array}$ & $\begin{array}{l}\text { 2- SB LL 4,0 } \\
\mathrm{kN}\end{array}$ & $\begin{array}{l}\text { - SB H.Adj. } \\
\text { Lower }\end{array}$ & $\begin{array}{l}\text { 3- SC Stroke } \\
\text { "c" }\end{array}$ & $\begin{array}{l}1225 \text { / } 297 \\
\text { (Up Front) }\end{array}$ & & \\
\hline Simulation 10 & $\begin{array}{c}2 \text {-DAB Vent } \\
\text { Holes } 2 \times \varnothing 15 \mathrm{~mm}\end{array}$ & $\begin{array}{c}2 \text { - DAB Tethers } 2 \times 338 \mathrm{~mm} \\
(3 \mathrm{~h} / 9 \mathrm{~h})+1 \times 332 \mathrm{~mm}(6 \mathrm{~h})\end{array}$ & $\begin{array}{c}\text { 2-SB LL } 4,0 \\
k N\end{array}$ & $\begin{array}{c}\text { 3- SB H.Adj. } \\
\text { Lower }\end{array}$ & $\begin{array}{l}\text { 3- SC Stroke } \\
\text { "c" }\end{array}$ & $\begin{array}{c}1243 / 279 \\
\text { (Down Rear) }\end{array}$ & $\begin{array}{l}\text { Nominal } \\
+0,5 \mathrm{~N}\end{array}$ & \\
\hline Simulation 11 & $\begin{array}{c}\text { 2-DAB Vent } \\
\text { Holes } 2 \times \varnothing 15 \mathrm{~mm}\end{array}$ & 3- DAB No tether & $\begin{array}{c}\text { 3-SB LL 3,0 } \\
k N\end{array}$ & $\begin{array}{c}\text { 1- SB H. Adj. } \\
\text { Upper }\end{array}$ & $\begin{array}{l}\text { 1- SC Stroke } \\
\text { "a" }\end{array}$ & $\begin{array}{l}1225 \text { / } 297 \\
\text { (Up Front) }\end{array}$ & $\begin{array}{l}\text { Nominal } \\
-0,5 \mathrm{~N}\end{array}$ & \\
\hline Simulation 12 & $\begin{array}{c}2 \text {-DAB Vent } \\
\text { Holes } 2 \times \varnothing 15 \mathrm{~mm}\end{array}$ & 3 - DAB No tether & $\begin{array}{l}\text { 3- SB LL } 3,0 \\
\mathrm{kN}\end{array}$ & $\begin{array}{l}1 \text { - SB H. Adj. } \\
\text { Upper }\end{array}$ & $\begin{array}{l}\text { 1- SC Stroke } \\
\text { "a" }\end{array}$ & $\begin{array}{c}1243 / 279 \\
\text { (Down Rear) }\end{array}$ & & \\
\hline Simulation 13 & $\begin{array}{c}\text { - DAB Vent } \\
\text { Holes } 2 \times ø 35 \mathrm{~mm}\end{array}$ & $\begin{array}{c}1 \text { - DAB Tethers } 2 \times 338 \mathrm{~mm} \\
(3 \mathrm{~h} / 9 \mathrm{~h})\end{array}$ & $\begin{array}{c}\text { 2-SB LL 4,0 } \\
k N\end{array}$ & $\begin{array}{c}\text { - SB H. Adj. } \\
\text { Upper }\end{array}$ & $\begin{array}{c}\text { 3- SC Stroke } \\
\text { "c" }\end{array}$ & $\begin{array}{l}1225 / 297 \\
\text { (Up Front) }\end{array}$ & $\begin{array}{c}\text { Nominal } \\
-0,5 \mathrm{~N}\end{array}$ & $\begin{array}{l}+700 \mathrm{~N} / \\
+1000 \mathrm{~N}\end{array}$ \\
\hline Simulation 14 & $\begin{array}{c}\text { 3- DAB Vent } \\
\text { Holes } 2 \times \varnothing 35 \mathrm{~mm}\end{array}$ & $\begin{array}{c}1 \text { - DAB Tethers } 2 \times 338 \mathrm{~mm} \\
(3 \mathrm{~h} / 9 \mathrm{~h})\end{array}$ & $\begin{array}{l}\text { 2- SB LL } 4,0 \\
\mathrm{kN}\end{array}$ & $\begin{array}{l}1 \text { - SB H. Adj. } \\
\text { Upper }\end{array}$ & $\begin{array}{l}\text { 3- SC Stroke } \\
\text { "c" }\end{array}$ & $\begin{array}{c}1243 / 279 \\
\text { (Down Rear) }\end{array}$ & $\begin{array}{l}\text { Nominal } \\
+0,5 \mathrm{~N}\end{array}$ & $\begin{array}{l}-700 \mathrm{~N} / \\
-1000 \mathrm{~N}\end{array}$ \\
\hline Simulation 15 & \begin{tabular}{|c|} 
- DAB Vent \\
Holes $2 \times \oslash 35 \mathrm{~mm}$
\end{tabular} & $\begin{array}{c}2 \text { - DAB Tethers } 2 \times 338 \mathrm{~mm} \\
(3 \mathrm{~h} / 9 \mathrm{~h})+1 \times 332 \mathrm{~mm}(6 \mathrm{~h})\end{array}$ & $\begin{array}{c}\text { 3- SB LL } 3,0 \\
\mathrm{kN}\end{array}$ & $\begin{array}{l}\text { 2- SB H.Adj. } \\
\text { Middle }\end{array}$ & $\begin{array}{l}1 \text { - SC Stroke } \\
\text { "a" }\end{array}$ & $\begin{array}{l}1225 / 297 \\
\text { (Up Front) }\end{array}$ & $\begin{array}{l}\text { Nominal } \\
-0,5 \mathrm{~N}\end{array}$ & \\
\hline Simulation 16 & $\begin{array}{c}3 \text { - DAB Vent } \\
\text { Holes } 2 \times ø 35 \mathrm{~mm}\end{array}$ & $\begin{array}{c}\text { 2- DAB Tethers } 2 \times 338 \mathrm{~mm} \\
(3 \mathrm{~h} / 9 \mathrm{~h})+1 \times 332 \mathrm{~mm}(6 \mathrm{~h})\end{array}$ & $\begin{array}{c}\text { 3- SB LL 3,0 } \\
\text { kN }\end{array}$ & $\begin{array}{l}\text { 2- SB H.Adj. } \\
\text { Middle }\end{array}$ & $\begin{array}{c}\text { 1- SC Stroke } \\
\text { "a" }\end{array}$ & $\begin{array}{c}1243 / 279 \\
\text { (Down Rear) }\end{array}$ & $\begin{array}{l}\text { Nominal } \\
+0,5 \mathrm{~N}\end{array}$ & $\begin{array}{l}-700 \mathrm{~N} / \\
-1000 \mathrm{~N} \\
\end{array}$ \\
\hline Simulation 17 & \begin{tabular}{|c|}
3 - DAB Vent \\
Holes $2 \times ø 35 \mathrm{~mm}$
\end{tabular} & 3 - DAB No tether & $\begin{array}{c}1 \text { - SB LL 3,0 } \\
\text { kN }\end{array}$ & $\begin{array}{c}3 \text { - SB H.Adj. } \\
\text { Lower }\end{array}$ & $\begin{array}{c}\text { 2- SC Stroke } \\
\text { "b" }\end{array}$ & $\begin{array}{l}1225 / 297 \\
\text { (Up Front) }\end{array}$ & $\begin{array}{c}\text { Nominal } \\
-0,5 \mathrm{~N}\end{array}$ & $\begin{array}{l}+700 \mathrm{~N} / \\
+1000 \mathrm{~N}\end{array}$ \\
\hline Simulation 18 & $\begin{array}{c}3 \text { - DAB Vent } \\
\text { Holes } 2 \times \varnothing 35 \mathrm{~mm}\end{array}$ & 3 - DAB No tether & $\begin{array}{c}1-\mathrm{SB} \mathrm{LL} \mathrm{3,0} \\
\mathrm{kN}\end{array}$ & $\begin{array}{l}\text { 3 - SB H.Adj. } \\
\text { Lower }\end{array}$ & $\begin{array}{c}\text { 2- SC Stroke } \\
\text { "b" }\end{array}$ & $\begin{array}{c}1243 / 279 \\
\text { (Down Rear) }\end{array}$ & $\begin{array}{l}\text { Nominal } \\
+0,5 \mathrm{~N}\end{array}$ & $\begin{array}{l}-700 \mathrm{~N} / \\
-1000 \mathrm{~N}\end{array}$ \\
\hline Simulation 19 & $\begin{array}{c}1 \text {-DAB Vent } \\
\text { Holes } 2 \times \varnothing 25 \mathrm{~mm}\end{array}$ & $\begin{array}{c}1 \text { - DAB Tethers } 2 \times 338 \mathrm{~mm} \\
(3 \mathrm{~h} / 9 \mathrm{~h})\end{array}$ & $\begin{array}{c}\text { 3- SB LL } 3,0 \\
k N\end{array}$ & $\begin{array}{l}\text { 3- SB H.Adj. } \\
\text { Lower }\end{array}$ & $\begin{array}{c}\text { 2- SC Stroke } \\
\text { "b" }\end{array}$ & $\begin{array}{l}1225 \text { / } 297 \\
\text { (Up Front) }\end{array}$ & $\begin{array}{l}\text { Nominal } \\
-0,5 \mathrm{~N}\end{array}$ & \\
\hline Simulation 20 & $\left|\begin{array}{c}1 \text {-DAB Vent } \\
\text { Holes } 2 \times \varnothing 25 \mathrm{~mm}\end{array}\right|$ & $\begin{array}{c}1 \text { - DAB Tethers } 2 \times 338 \mathrm{~mm} \\
(3 \mathrm{~h} / 9 \mathrm{~h})\end{array}$ & $\begin{array}{c}\text { 3- SB LL 3,0 } \\
\text { kN }\end{array}$ & $\begin{array}{c}\text { - SB H.Adj. } \\
\text { Lower }\end{array}$ & $\begin{array}{l}\text { 2- SC Stroke } \\
\text { "b" }\end{array}$ & $\begin{array}{c}1243 / 279 \\
\text { (Down Rear) }\end{array}$ & $\begin{array}{l}\text { Nominal } \\
+0,5 \mathrm{~N}\end{array}$ & $\begin{array}{l}-700 \mathrm{~N} / \\
-1000 \mathrm{~N}\end{array}$ \\
\hline
\end{tabular}




\begin{tabular}{|c|c|c|c|c|c|c|c|c|}
\hline Simulation 21 & $\begin{array}{c}\text { 1-DAB Vent } \\
\text { Holes } 2 \times \varnothing 25 \mathrm{~mm}\end{array}$ & $\begin{array}{l}2 \text { - DAB Tethers } 2 \times 338 \mathrm{~mm} \\
(3 \mathrm{~h} / 9 \mathrm{~h})+1 \times 332 \mathrm{~mm}(6 \mathrm{~h})\end{array}$ & $\begin{array}{c}\text { 1- SB LL 3,0 } \\
\mathrm{kN}\end{array}$ & $\begin{array}{l}1 \text { - SB H. Adj. } \\
\text { Upper }\end{array}$ & $\begin{array}{c}\text { 3- SC Stroke } \\
\text { "c" }\end{array}$ & $\begin{array}{l}1225 \text { / } 297 \\
\text { (Up Front) }\end{array}$ & $\begin{array}{l}\text { Nominal } \\
-0,5 \mathrm{~N}\end{array}$ & $\begin{array}{l}+700 \mathrm{~N} / \\
+1000 \mathrm{~N}\end{array}$ \\
\hline Simulation 22 & $\begin{array}{c}1 \text {-DAB Vent } \\
\text { Holes } 2 \times \varnothing 25 \mathrm{~mm}\end{array}$ & $\begin{array}{l}\text { 2- DAB Tethers } 2 \times 338 \mathrm{~mm} \\
(3 \mathrm{~h} / 9 \mathrm{~h})+1 \times 332 \mathrm{~mm}(6 \mathrm{~h})\end{array}$ & $\begin{array}{c}1 \text { - SB LL 3,0 } \\
\mathrm{kN}\end{array}$ & $\begin{array}{l}1 \text { - SB H. Adj. } \\
\text { Upper }\end{array}$ & $\begin{array}{l}\text { 3- SC Stroke } \\
\text { "c" }\end{array}$ & $\begin{array}{l}1243 / 279 \\
\text { (Down Rear) }\end{array}$ & & \\
\hline Simulation 23 & $\begin{array}{c}1 \text {-DAB Vent } \\
\text { Holes } 2 \times \varnothing 25 \mathrm{~mm}\end{array}$ & 3 - DAB No tether & $\begin{array}{c}\text { 2-SB LL 4,0 } \\
\text { kN }\end{array}$ & $\begin{array}{l}\text { 2- SB H.Adj. } \\
\text { Middle }\end{array}$ & $\begin{array}{c}1 \text { - SC Stroke } \\
\text { "a" }\end{array}$ & $\begin{array}{l}1225 \text { / } 297 \\
\text { (Up Front) }\end{array}$ & & \\
\hline Simulation 24 & $\begin{array}{c}1 \text {-DAB Vent } \\
\text { Holes } 2 \times \varnothing 25 \mathrm{~mm}\end{array}$ & 3 - DAB No tether & $\begin{array}{c}\text { 2- SB LL 4,0 } \\
k N\end{array}$ & $\begin{array}{l}\text { 2- SB H.Adj. } \\
\text { Middle }\end{array}$ & $\begin{array}{c}\text { 1- SC Stroke } \\
\text { "a" }\end{array}$ & $\begin{array}{c}1243 / 279 \\
\text { (Down Rear) }\end{array}$ & $\begin{array}{l}\text { Nominal } \\
+0,5 \mathrm{~N}\end{array}$ & \\
\hline Simulation 25 & $\begin{array}{c}2 \text {-DAB Vent } \\
\text { Holes } 2 \times \varnothing 15 \mathrm{~mm}\end{array}$ & $\begin{array}{c}1 \text { - DAB Tethers } 2 \times 338 \mathrm{~mm} \\
(3 \mathrm{~h} / 9 \mathrm{~h})\end{array}$ & $\begin{array}{c}\text { 2-SB LL 4,0 } \\
\text { kN }\end{array}$ & $\begin{array}{l}\text { 3- SB H.Adj. } \\
\text { Lower }\end{array}$ & $\begin{array}{c}1 \text { - SC Stroke } \\
\text { "a" }\end{array}$ & $\begin{array}{l}1225 \text { / } 297 \\
\text { (Up Front) }\end{array}$ & $\begin{array}{c}\text { Nominal } \\
-0,5 \mathrm{~N} \\
\end{array}$ & $\begin{array}{l}+700 \mathrm{~N} / \\
+1000 \mathrm{~N}\end{array}$ \\
\hline Simulation 26 & $\begin{array}{c}2 \text {-DAB Vent } \\
\text { Holes } 2 \times \varnothing 15 \mathrm{~mm}\end{array}$ & $\begin{array}{c}1 \text { - DAB Tethers } 2 \times 338 \mathrm{~mm} \\
(3 \mathrm{~h} / 9 \mathrm{~h})\end{array}$ & $\begin{array}{c}\text { 2-SB LL 4,0 } \\
\text { kN }\end{array}$ & $\begin{array}{l}\text { 3- SB H.Adj. } \\
\text { Lower }\end{array}$ & $\begin{array}{c}1 \text { - SC Stroke } \\
\text { "a" }\end{array}$ & $\begin{array}{c}1243 / 279 \\
\text { (Down Rear) }\end{array}$ & & \\
\hline Simulation 27 & $\begin{array}{c}2 \text {-DAB Vent } \\
\text { Holes } 2 \times \varnothing 15 \mathrm{~mm}\end{array}$ & $\begin{array}{l}\text { 2- DAB Tethers } 2 \times 338 \mathrm{~mm} \\
(3 \mathrm{~h} / 9 \mathrm{~h})+1 \times 332 \mathrm{~mm}(6 \mathrm{~h})\end{array}$ & $\begin{array}{c}\text { 3- SB LL 3,0 } \\
k N\end{array}$ & $\begin{array}{c}\text { 1- SB H. Adj. } \\
\text { Upper }\end{array}$ & $\begin{array}{c}\text { 2- SC Stroke } \\
\text { "b" }\end{array}$ & $\begin{array}{l}1225 / 297 \\
\text { (Up Front) } \\
\end{array}$ & $\begin{array}{l}\text { Nominal } \\
-0,5 \mathrm{~N}\end{array}$ & $\begin{array}{l}+700 \mathrm{~N} / \\
+1000 \mathrm{~N} \\
\end{array}$ \\
\hline Simulation 28 & $\begin{array}{c}2 \text {-DAB Vent } \\
\text { Holes } 2 \times \varnothing 15 \mathrm{~mm}\end{array}$ & $\begin{array}{l}2-\text { DAB Tethers } 2 \times 338 \mathrm{~mm} \\
(3 \mathrm{~h} / 9 \mathrm{~h})+1 \times 332 \mathrm{~mm}(6 \mathrm{~h})\end{array}$ & $\begin{array}{c}3 \text { - SB LL } 3,0 \\
\mathrm{kN}\end{array}$ & $\begin{array}{c}1 \text { - SB H. Adj. } \\
\text { Upper }\end{array}$ & $\begin{array}{c}\text { 2- SC Stroke } \\
\text { "b" }\end{array}$ & $\begin{array}{c}1243 / 279 \\
\text { (Down Rear) }\end{array}$ & $\begin{array}{c}\text { Nominal } \\
+0,5 \mathrm{~N} \\
\end{array}$ & $\begin{array}{l}-700 \mathrm{~N} / \\
-1000 \mathrm{~N} \\
\end{array}$ \\
\hline Simulation 29 & $\begin{array}{c}2 \text {-DAB Vent } \\
\text { Holes } 2 \times \varnothing 15 \mathrm{~mm}\end{array}$ & 3 - DAB No tether & $\begin{array}{c}1 \text { - SB LL } 3,0 \\
k N\end{array}$ & $\begin{array}{l}\text { 2- SB H.Adj. } \\
\text { Middle }\end{array}$ & $\begin{array}{c}\text { 3- SC Stroke } \\
\text { "c" }\end{array}$ & $\begin{array}{l}1225 / 297 \\
\text { (Up Front) }\end{array}$ & & \\
\hline Simulation 30 & $\begin{array}{c}2 \text {-DAB Vent } \\
\text { Holes } 2 \times \varnothing 15 \mathrm{~mm}\end{array}$ & 3- DAB No tether & $\begin{array}{c}1 \text { - SB LL 3,0 } \\
\text { kN }\end{array}$ & $\begin{array}{l}\text { 2- SB H.Adj. } \\
\text { Middle }\end{array}$ & $\begin{array}{c}\text { 3- SC Stroke } \\
\text { "c" }\end{array}$ & $\begin{array}{c}1243 / 279 \\
\text { (Down Rear) }\end{array}$ & & \\
\hline Simulation 31 & $\begin{array}{c}3 \text { - DAB Vent } \\
\text { Holes } 2 \times \varnothing 35 \mathrm{~mm}\end{array}$ & $\begin{array}{c}1 \text { - DAB Tethers } 2 \times 338 \mathrm{~mm} \\
(3 \mathrm{~h} / 9 \mathrm{~h})\end{array}$ & $\begin{array}{c}3-\mathrm{SB} \text { LL } 3,0 \\
\mathrm{kN}\end{array}$ & $\begin{array}{l}\text { 2- SB H.Adj. } \\
\text { Middle }\end{array}$ & $\begin{array}{c}\text { 3- SC Stroke } \\
\text { "c" }\end{array}$ & $\begin{array}{l}1225 \text { / } 297 \\
\text { (Up Front) }\end{array}$ & $\begin{array}{l}\text { Nominal } \\
-0,5 \mathrm{~N}\end{array}$ & \\
\hline Simulation 32 & $\begin{array}{c}3 \text { - DAB Vent } \\
\text { Holes } 2 \times \varnothing 35 \mathrm{~mm}\end{array}$ & $\begin{array}{c}1 \text { - DAB Tethers } 2 \times 338 \mathrm{~mm} \\
(3 \mathrm{~h} / 9 \mathrm{~h})\end{array}$ & $\begin{array}{c}\text { 3- SB LL 3,0 } \\
\mathrm{kN}\end{array}$ & $\begin{array}{l}\text { 2- SB H.Adj. } \\
\text { Middle }\end{array}$ & $\begin{array}{l}\text { 3- SC Stroke } \\
\text { "c" }\end{array}$ & $\begin{array}{l}1243 / 279 \\
\text { (Down Rear) }\end{array}$ & $\begin{array}{l}\text { Nominal } \\
+0,5 \mathrm{~N}\end{array}$ & \\
\hline Simulation 33 & $\begin{array}{c}3 \text { - DAB Vent } \\
\text { Holes } 2 \times \varnothing 35 \mathrm{~mm}\end{array}$ & $\begin{array}{l}2 \text { - DAB Tethers } 2 \times 338 \mathrm{~mm} \\
(3 \mathrm{~h} / 9 \mathrm{~h})+1 \times 332 \mathrm{~mm}(6 \mathrm{~h})\end{array}$ & $\begin{array}{c}1 \text { - SB LL 3,0 } \\
\text { kN }\end{array}$ & $\begin{array}{l}\text { 3-SB H.Adj. } \\
\text { Lower }\end{array}$ & $\begin{array}{l}\text { 1- SC Stroke } \\
\text { "a" }\end{array}$ & $\begin{array}{l}1225 / 297 \\
\text { (Up Front) }\end{array}$ & $\begin{array}{l}\text { Nominal } \\
-0,5 \mathrm{~N}\end{array}$ & $\begin{array}{l}+700 \mathrm{~N} / \\
+1000 \mathrm{~N}\end{array}$ \\
\hline Simulation 34 & $\begin{array}{c}3 \text { - DAB Vent } \\
\text { Holes } 2 \times \varnothing 35 \mathrm{~mm}\end{array}$ & $\begin{array}{l}\text { 2- DAB Tethers } 2 \times 338 \mathrm{~mm} \\
(3 \mathrm{~h} / 9 \mathrm{~h})+1 \times 332 \mathrm{~mm}(6 \mathrm{~h})\end{array}$ & $\begin{array}{c}1 \text { - SB LL 3,0 } \\
\text { kN }\end{array}$ & $\begin{array}{l}3 \text { - SB H.Adj. } \\
\text { Lower }\end{array}$ & $\begin{array}{c}1 \text { - SC Stroke } \\
\text { "a" }\end{array}$ & $\begin{array}{l}1243 / 279 \\
\text { (Down Rear) }\end{array}$ & $\begin{array}{l}\text { Nominal } \\
+0,5 \mathrm{~N}\end{array}$ & $\begin{array}{l}-700 \mathrm{~N} / \\
-1000 \mathrm{~N}\end{array}$ \\
\hline Simulation 35 & $\begin{array}{c}3 \text { - DAB Vent } \\
\text { Holes } 2 \times \varnothing 35 \mathrm{~mm}\end{array}$ & 3- DAB No tether & $\begin{array}{c}\text { 2-SB LL 4,0 } \\
k N\end{array}$ & $\begin{array}{l}\text { 1- SB H. Adj. } \\
\text { Upper }\end{array}$ & $\begin{array}{c}\text { 2- SC Stroke } \\
\text { "b" }\end{array}$ & $\begin{array}{l}1225 / 297 \\
\text { (Up Front) }\end{array}$ & & \\
\hline Simulation 36 & $\begin{array}{c}3 \text { - DAB Vent } \\
\text { Holes } 2 \times \varnothing 35 \mathrm{~mm}\end{array}$ & 3 - DAB No tether & $\begin{array}{l}\text { 2- SB LL } 4,0 \\
\mathrm{kN}\end{array}$ & $\begin{array}{l}\text { 1- SB H. Adj. } \\
\text { Upper }\end{array}$ & $\begin{array}{l}\text { 2- SC Stroke } \\
\text { "b" }\end{array}$ & $\begin{array}{c}1243 / 279 \\
\text { (Down Rear) }\end{array}$ & $\begin{array}{l}\text { Nominal } \\
+0,5 \mathrm{~N}\end{array}$ & $\begin{array}{l}-700 \mathrm{~N} / \\
-1000 \mathrm{~N}\end{array}$ \\
\hline
\end{tabular}

As mentioned before, even though the main focus is developing a driver restraint system with the lowest chest compression as possible, all biomechanical responses like head, neck, femur, tibia and knee were assessed in accordance Brazilian Legal Requirement.

\section{Conduct Data Analysis}

For all 36 runs were identified the maximum and minimum values for each injury criteria. Therefore, Table 3 contains a summary of all injuries evaluated.

Table 3. Summary results for all injuries criteria.

\begin{tabular}{|c|c|c|c|c|c|}
\hline Dummy Region & Injury Criteria & Units & Values & $\begin{array}{c}\text { MIN } \\
\text { Value }\end{array}$ & $\begin{array}{l}\text { MAX } \\
\text { Value }\end{array}$ \\
\hline Head & $\mathrm{HIC}(36 \mathrm{~ms})$ & {$[-]$} & 1000 & OK & OK \\
\hline \multirow{8}{*}{ Neck } & Resultant Acceleration (3 ms) & [g] & 80 & OK & $\mathrm{OK}$ \\
\hline & \multirow{3}{*}{ Axial Tension $(+F z)$ - Duration } & \multirow{3}{*}[N]{} & $3300(0 \mathrm{~ms})$ & OK & $\mathrm{OK}$ \\
\hline & & & $2900(35 \mathrm{~ms})$ & OK & $\mathrm{OK}$ \\
\hline & & & $1100(\geq 60 \mathrm{~ms})$ & OK & OK \\
\hline & \multirow{3}{*}{ Shear Load (+Fx) - Duration } & \multirow{3}{*}[N]{} & $3100(0 \mathrm{~ms})$ & OK & $\mathrm{OK}$ \\
\hline & & & $1500(25-35 \mathrm{~ms})$ & $\mathrm{OK}$ & $\mathrm{OK}$ \\
\hline & & & $1100(\geq 45 \mathrm{~ms})$ & OK & $\mathrm{OK}$ \\
\hline & Rearward Moment - Ext (-My) & {$[\mathrm{Nm}]$} & 57 & OK & $\mathrm{OK}$ \\
\hline \multirow{2}{*}{ Thorax } & Compression (Belted) & {$[\mathrm{mm}]$} & 50 & $\mathrm{OK}$ & NOK \\
\hline & Viscous Criterion & {$[\mathrm{m} / \mathrm{s}]$} & 1 & $\mathrm{OK}$ & $\mathrm{OK}$ \\
\hline \multirow{2}{*}{ Femur } & \multirow{2}{*}{ Compressive Load - Duration } & \multirow{2}{*}[N]{} & $9070(0 \mathrm{~ms})$ & OK & $\mathrm{OK}$ \\
\hline & & & $7580(0 \mathrm{~ms})$ & OK & $\mathrm{OK}$ \\
\hline \multirow{2}{*}{ Tibia } & Axial Compressive Load & {$[\mathrm{N}]$} & 8000 & OK & $\mathrm{OK}$ \\
\hline & Index $=\mathrm{M} / \mathrm{Mc}+|\mathrm{P}| / \mathrm{Pc}$ & {$[-]$} & 1.3 & OK & OK \\
\hline Knee & Slidding (Tibia-Femur Translation) & {$[\mathrm{mm}]$} & 15 & OK & $\mathrm{OK}$ \\
\hline
\end{tabular}

The cells filled in red in the table indicate that the legal requirement was not fulfilled for a specific combination of simulation; the cells filled in yellow indicate that the 
legal requirement was fulfilled however are not lower than a pre-determined safety margin and the cells filled in green in the table indicate that the legal requirement and pre-determined safety margin were fulfilled.

Table 4 shows if the chest compression fulfilled the legal requirement and safety margin. As we can verify in the table, the chest compression is below the safety margin for 3 combinations (cells filled in green), for 29 combinations the chest compression is above the safety margin but below the legal requirement (cells filled in yellow), and for 4 combination the chest compression is above the legal requirement (cells filled in red).

Table 4. Chest compression results to all simulations performed.

\begin{tabular}{|l|l|}
\hline Simulation 1 & \\
\hline Simulation 2 & \\
\hline Simulation 3 & \\
\hline Simulation 4 & \\
\hline Simulation 5 & \\
\hline Simulation 6 & \\
\hline Simulation 7 & \\
\hline Simulation 8 & \\
\hline Simulation 9 & \\
\hline
\end{tabular}
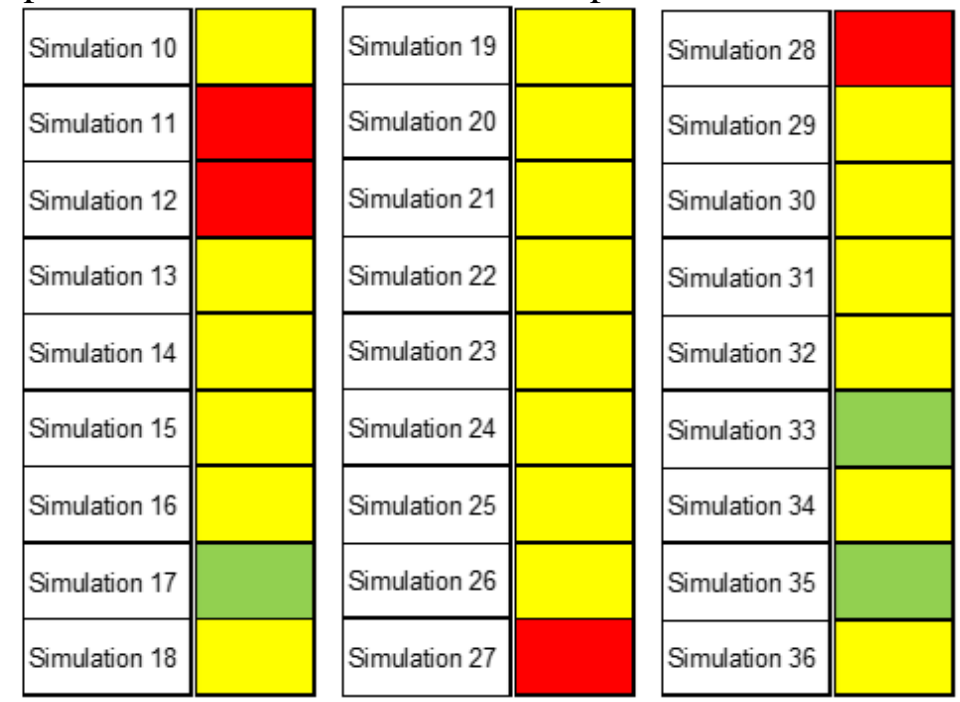

The Figures 20 and 21 are based on the Signal to Noise Ratio (S/N ratio) which represents the robustness of each parameter and mean square deviation which shows the efficiency, respectively.

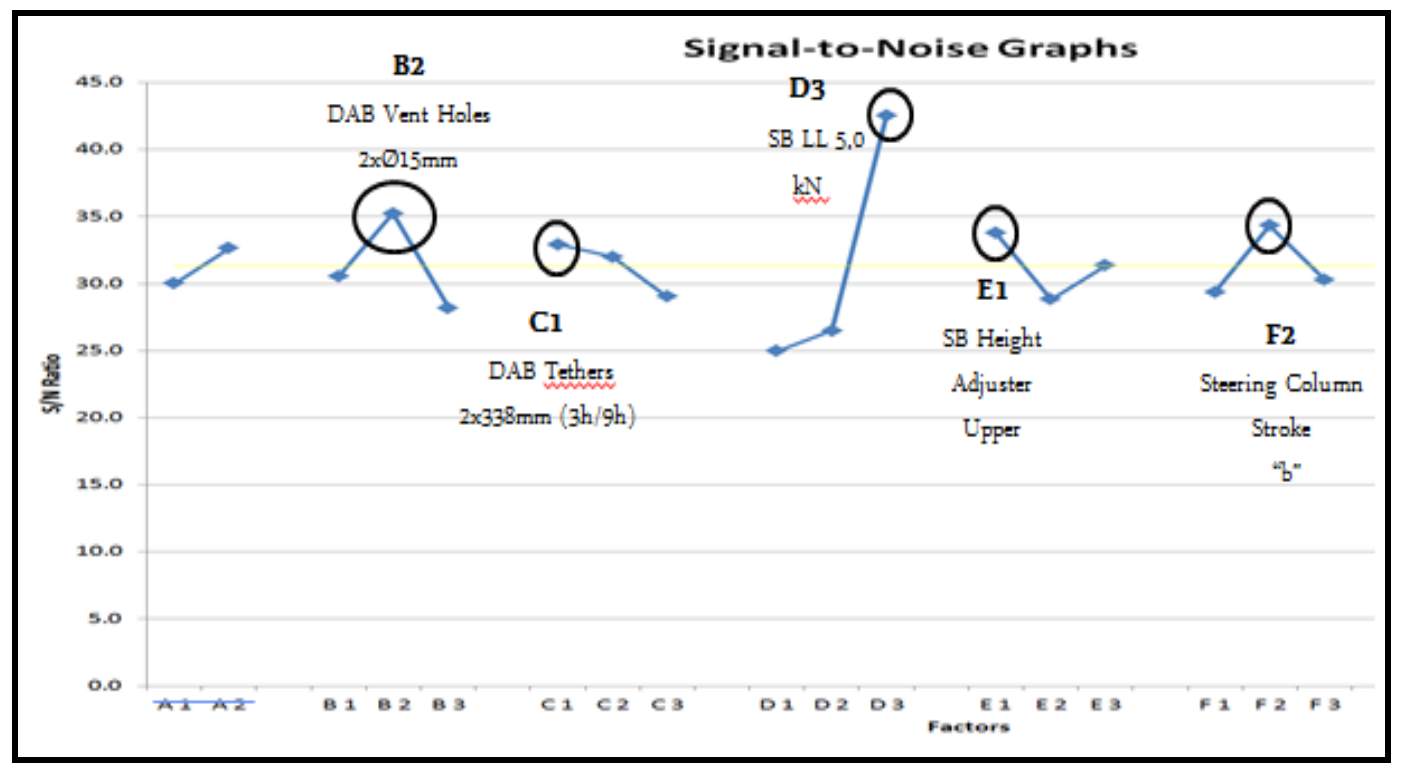

Figure 20. S/N to chest compression. 


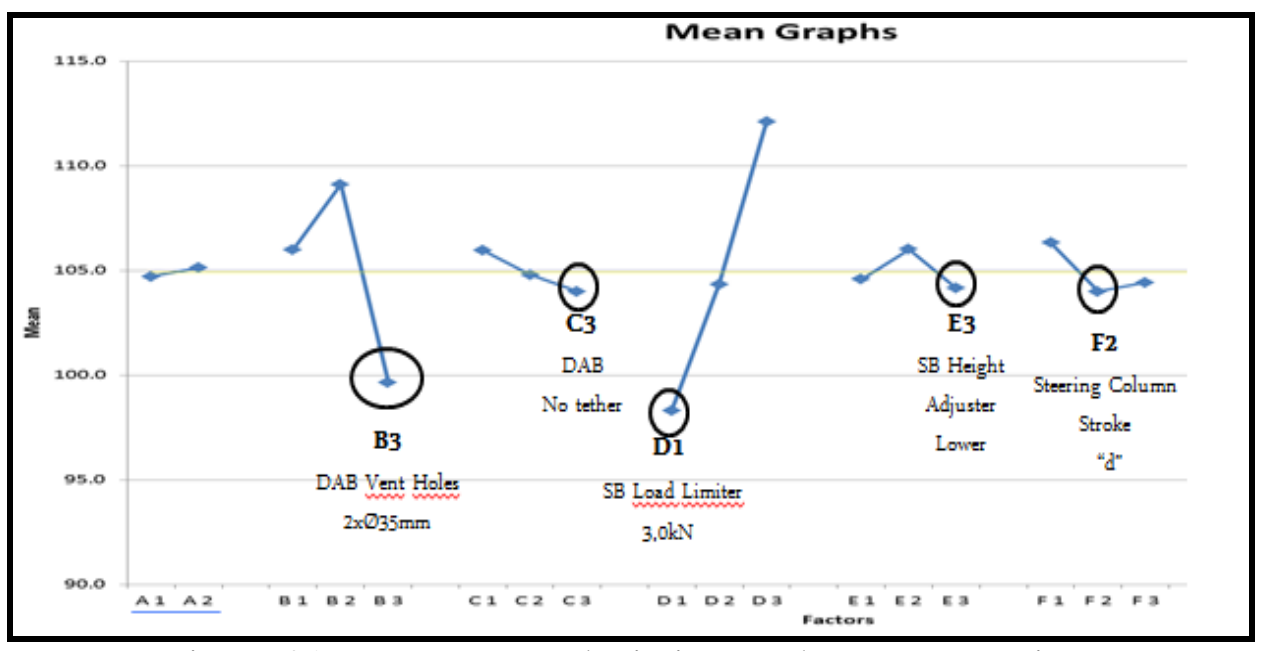

Figure 21. Mean square deviation to chest compression.

Analyzing the Figures 20 and 21, for some control factors the highest S/N did not meet the safety margin with respect to chest compression. Therefore, the team decided to run additional numerical simulations in order to verify the best combination. The first combination was performed considering the highest $\mathrm{S} / \mathrm{N}$, but lower than safety margin. Second combination considered the lowest mean square deviation (MSD), see Table 5.

Table 5. Confirmation run considering combinations with highest $\mathrm{S} / \mathrm{N}$ and lower MSD.

\begin{tabular}{|c|c|c|c|c|c|c|c|c|c|}
\hline & & \multicolumn{5}{|c|}{ CONTROL FACTORS } & \multicolumn{3}{|c|}{ Noise Factor } \\
\hline & & $\begin{array}{c}\text { B } \\
\text { DAB Vent Hole }\end{array}$ & $\begin{array}{c}\mathrm{C} \\
\text { DAB Tether }\end{array}$ & \begin{tabular}{|c|} 
D \\
SBLood Limiter \\
\end{tabular} & $\begin{array}{c}\text { E } \\
\text { SB High Adi. }\end{array}$ & \begin{tabular}{|c|}
$\mathrm{F}$ \\
s. Column Stroke \\
\end{tabular} & \begin{tabular}{|c|} 
ATD Position (H \\
point tzlz
\end{tabular} & $\begin{array}{c}\text { Load Limiter } \\
\text { Tolef ance }\end{array}$ & $\begin{array}{l}\text { St Column Saroke Tol. } \\
\text { (Inicial Peak Loadd } \\
\text { Punning Lood]l] }\end{array}$ \\
\hline \multirow{2}{*}{ 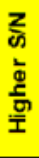 } & $\mathbf{M n}$ & $\begin{array}{c}3 \text { - DAB Vent Holes } \\
2 \times Ø 35 \mathrm{~mm}\end{array}$ & $\begin{array}{l}1 \text { - DAB Tethers } \\
2 \times 338 \mathrm{~mm} \text { (3h/9h) }\end{array}$ & $1-\mathrm{SBLL} 3.0 \mathrm{kN}$ & $\begin{array}{c}1 \text { - SB H. Adj. } \\
\text { Upper }\end{array}$ & 2-SC Stroke " " " & $\begin{array}{l}1225 / 297 \\
\text { (Up Front) }\end{array}$ & $\begin{array}{l}\text { Nominal } \\
-0,5 \mathrm{~N}\end{array}$ & $\begin{array}{l}+700 \mathrm{~N} / \\
+1000 \mathrm{~N}\end{array}$ \\
\hline & N2 & $\begin{array}{c}3-\mathrm{DAB} \text { Vent Holes } \\
2 \times \oslash 35 \mathrm{~mm}\end{array}$ & $\begin{array}{l}1 \text { - DAB Tethers } \\
2 \times 338 \mathrm{~mm} \text { (3h/9h) }\end{array}$ & $1-S B L L 3.0 \mathrm{kN}$ & $\begin{array}{c}1 \text { - SB H. Adj. } \\
\text { Upper }\end{array}$ & 2-SC Stroke "b" & $\begin{array}{c}1243 / 279 \\
\text { (Down Rear) }\end{array}$ & $\begin{array}{r}\text { Nominal } \\
+0,5 \mathrm{~N} \\
\end{array}$ & $\begin{array}{l}-700 \mathrm{~N} / \\
-1000 \mathrm{~N}\end{array}$ \\
\hline \multirow{2}{*}{ 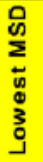 } & NI & $\begin{array}{c}3 \text { - DAB Vent Holes } \\
2 \times 035 \mathrm{~mm}\end{array}$ & 3-DAB No tether & $1-\mathrm{SBLL} 3.0 \mathrm{kN}$ & $\begin{array}{l}\text { 3-SB HAd: } \\
\text { Lower }\end{array}$ & 2- SC Stroke " "b" & $\begin{array}{l}1225 / 297 \\
\text { (Up Front) }\end{array}$ & $\begin{array}{r}\text { Nominal } \\
-0,5 \mathrm{~N} \\
\end{array}$ & $\begin{array}{l}+700 \mathrm{~N} / \\
+1000 \mathrm{~N} \\
\end{array}$ \\
\hline & N2 & $\begin{array}{c}\text { 3- DAB Vent Holes } \\
2 \times \oslash 35 \mathrm{~mm}\end{array}$ & 3-DAB No tether & $1-\mathrm{SBLL} 3.0 \mathrm{kN}$ & $\begin{array}{l}\text { 3-SB HAdi. } \\
\text { Lower }\end{array}$ & 2-SC Stroke "b" & $\begin{array}{c}1243 / 279 \\
\text { (Down Rear) }\end{array}$ & $\begin{array}{l}\text { Nominal } \\
+0,5 \mathrm{~N}\end{array}$ & $\begin{array}{l}-700 \mathrm{~N} / \\
-1000 \mathrm{~N}\end{array}$ \\
\hline
\end{tabular}

Analyzing the results, Table 6 , we verified that both combinations met the chest compression legal requirement. However, they are few above the safety margin (inhouse development target) for one of combination of noise. Therefore, the team decided to consider the combination with the lowest MSD as the best combination because it presented the lower variation.

Table 6. Summary results considering combinations with highest $\mathrm{S} / \mathrm{N}$ and lower MSD.

\begin{tabular}{|c|c|}
\hline & $\begin{array}{l}\% \text { of safety } \\
\text { margin }\end{array}$ \\
\hline \multirow{2}{*}{ 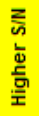 } & 97 \\
\hline & 107 \\
\hline \multirow{2}{*}{ 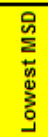 } & 94 \\
\hline & 103 \\
\hline
\end{tabular}




\section{Predict and Confirm}

According to rational presented and based on the graphics analyses the Table 7 shows the optimized combinations for chest compression, cells filled in green.

Table 7. Optimized combination.

\begin{tabular}{|c|c|c|c|c|}
\hline \multicolumn{2}{|c|}{ Control Factor } & $\begin{array}{ll}\text { or } & \text { Level } 1\end{array}$ & Level 2 & \multirow{2}{*}{$\begin{array}{r}\text { Level } 3 \\
2 \times \varnothing 35 \mathrm{mn}\end{array}$} \\
\hline B & $\begin{array}{l}\text { DAB Vent } \\
\text { Holes }\end{array}$ & $2 \times \varnothing 25 \mathrm{~mm}$ & $2 \times \varnothing 15 \mathrm{~mm}$ & \\
\hline C & $\begin{array}{l}\text { DAB Tethers } \\
\text { length }\end{array}$ & \begin{tabular}{c|c|}
$2 \times 338 \mathrm{~mm}$ \\
$(3 \mathrm{~h} / 9 \mathrm{~h})$
\end{tabular} & $\begin{array}{c}2 \times 338 \mathrm{~mm} \\
(3 \mathrm{~h} / 9 \mathrm{~h})+ \\
1 \times 332 \mathrm{~mm}(6 \mathrm{~h})\end{array}$ & No \\
\hline D & $\begin{array}{l}\text { SB Load } \\
\text { Limiter }\end{array}$ & $3,0 \mathrm{KN}$ & $4,0 \mathrm{KN}$ & $5,0 \mathrm{kN}$ \\
\hline $\mathrm{E}$ & $\begin{array}{l}\text { SB high } \\
\text { adjuster }\end{array}$ & Upper & Middle & Lower \\
\hline $\mathrm{F}$ & $=\begin{array}{l}\text { Steering } \\
\text { column Stroke }\end{array}$ & $\mathrm{ke}$ & b & c \\
\hline \multicolumn{5}{|c|}{ Running Load } \\
\hline & a & $4,700 \mathrm{~N}$ & \multicolumn{2}{|c|}{$4,800 \mathrm{~N}$} \\
\hline & b & $3,700 \mathrm{~N}$ & \multicolumn{2}{|c|}{$3,800 \mathrm{~N}$} \\
\hline & c & $3,000 \mathrm{~N}$ & \multicolumn{2}{|c|}{$3,000 \mathrm{~N}$} \\
\hline
\end{tabular}

To confirm the predicted parameters optimized, additional computational numerical simulations were performed to assess all injuries criterion considering the noise factors, Table 8.

Table 8. Summary results for all injuries criteria to optimized combination.

\begin{tabular}{|c|c|c|c|c|c|}
\hline \multirow{2}{*}{ Dummy Region } & \multirow[b]{2}{*}{ Injury Criteria } & \multicolumn{2}{|c|}{ 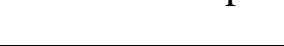 } & \multicolumn{2}{|c|}{ Lowest MSD } \\
\hline & & Units & Values & N1 & N2 \\
\hline Head & $\mathrm{HIC}(36 \mathrm{~ms})$ & {$[-]$} & 1000 & $\mathrm{OK}$ & $\mathrm{OK}$ \\
\hline \multirow{8}{*}{ Neck } & Resultant Acceleration (3 ms) & [g] & 80 & $\mathrm{OK}$ & $\mathrm{OK}$ \\
\hline & \multirow{3}{*}{ Axial Tension $(+F z)$ - Duration } & \multirow{3}{*}[N]{} & $3300(0 \mathrm{~ms})$ & $\mathrm{OK}$ & OK \\
\hline & & & 2900 (35 ms) & $\mathrm{OK}$ & $\mathrm{OK}$ \\
\hline & & & $1100(\geq 60 \mathrm{~ms})$ & OK & OK \\
\hline & \multirow{3}{*}{ Shear Load $(+\mathrm{Fx})$ - Duration } & \multirow{3}{*}[\mathrm{N}]{} & $3100(0 \mathrm{~ms})$ & OK & OK \\
\hline & & & $1500(25-35 \mathrm{~ms})$ & $\mathrm{OK}$ & $\mathrm{OK}$ \\
\hline & & & $1100(\geq 45 \mathrm{~ms})$ & OK & $\mathrm{OK}$ \\
\hline & Rearward Moment - Ext (-My) & {$[\mathrm{Nm}]$} & 57 & $\mathrm{OK}$ & $\mathrm{OK}$ \\
\hline \multirow{2}{*}{ Thorax } & Compression (Belted) & {$[\mathrm{mm}]$} & 50 & OK & $\mathrm{OK}$ \\
\hline & Viscous Criterion & {$[\mathrm{m} / \mathrm{s}]$} & 1 & $\mathrm{OK}$ & OK \\
\hline \multirow{2}{*}{ Femur } & \multirow{2}{*}{ Compressive Load - Duration } & \multirow{2}{*}[N]{} & $9070(0 \mathrm{~ms})$ & $\mathrm{OK}$ & $\mathrm{OK}$ \\
\hline & & & $7580(0 \mathrm{~ms})$ & OK & $\mathrm{OK}$ \\
\hline \multirow{2}{*}{ Tibia } & Axial Compressive Load & {$[\mathrm{N}]$} & 8000 & OK & $\mathrm{OK}$ \\
\hline & Index $=\mathrm{M} / \mathrm{Mc}+|\mathrm{P}| / \mathrm{Pc}$ & {$[-]$} & 1.3 & $\mathrm{OK}$ & $\mathrm{OK}$ \\
\hline Knee & Slidding (Tibia-Femur Translation) & {$[\mathrm{mm}]$} & 15 & $\mathrm{OK}$ & $\mathrm{OK}$ \\
\hline
\end{tabular}

Table 8 shows summary results for all injuries criteria. The cells filled in green indicate that the legal requirement was fulfilled; the cells filled in yellow indicate that the legal requirement was fulfilled, however the value is a few above the predetermined safety margin. 
So, the main focus of this project was achieved because the optimized combination presented injuries values below than regulatory requirements for all body regions.

\section{Document and Verify}

According to simulation results the optimized combination maintained the same performance than current baseline model and met the chest compression target for this project.

\subsection{IDDOV - Validate}

Until the moment the team did not perform the physical test to confirm the simulation results that indicates a reduction of chest compression injury using the optimized parameters, but based on another developments and previous correlation of the computational numerical model with the physical test, the team has confidence in the numerical results. Furthermore, the optimized combination was simulated by finite elements and confirms the improvement in all injuries criteria.

\section{CONCLUSIONS}

The optimized combination presents injuries values below than regulatory requirements for all body regions.

The optimized combination meets the project expected outcome for a Robust restraint system (without Safety Pretention) capable to keep the performance of CONTRAN 221/07 Legal Requirement (56 km/h 40\% ODB LHS) while saving mass and cost.

The optimized combination was simulated and showed a significant reduction at probability of injuries in a frontal impact according to CONTRAN 221/07. The computational numerical optimization tool helped to reduce the cost and time development of a safer vehicle which satisfies the current Brazilian regulations. The results presented excellent correlation between numerical simulation and physical test and the goals of the optimization were achieved showing that this tool is reliable and helpful for current and future developments.

\section{REFERENCES}

[1] Konrad Reif, "Fundamentals of Automotive and Engine Technology", 2014.

[2] Daw Alwerfalli and Trevor Lash, "Design For Six Sigma (DFSS) as a Proactive Business Process", Proceedings of the 2012 International Conference on Industrial Engineering and Operations Management Istanbul, Turkey, July 3-6, 2012.

[3] Myer Kutz, "Mechanical Engineers' Handbook: Materials and Mechanical Design", Volume 1, Third Edition, 2006.

[4] Taguchi, G., Chowdhury, S., and Wu, Y., “TAGUCHI'S Quality Engineering Handbook”, John Wiley \& Sons, Inc., Hoboken, NJ, 2004. 
[5] Zillur Rahman and Faisal Talib, "A Study of Optimization of Process by Using Taguchi's Parameter Design Approach", The Icfai University Journal of Operations Management, Vol. VII, No. 3, 2008.

[6] MADYMO Theory Manual. Release 6.4.1 December 2007.

\section{ACKNOWLEDGMENTS}

The authors wish to acknowledge the engineers and technicians at General Motors Brazil, for their help in developing and conducting the numerical computational simulations, analyzes and tests.

\section{RECOMMENDED FUTURE WORK}

Additional verification with this optimized combination across other restraint performance considerations should be performed to make sure the system is properly balanced. 